\title{
MISR research-aerosol-algorithm refinements for dark water retrievals
}

\author{
J. A. Limbacher and R. A. Kahn
}

Correspondence to: R. A. Kahn (ralph.kahn@nasa.gov) 


\section{Real Refractive Index $\left(n_{r}\right)$ Sensitivity Study}

\section{[Figures S1 - S5]}

This section expands on the AOD retrieval sensitivity analysis for the real refractive index $\left(n_{r}\right)$, illustrated in Figure 6 of the main paper. It covers a range of particle sizes, and $n_{r}$ as well as AOD values are varied systematically for the particles assumed in the algorithm comparison space.

For each figure, the simulated atmosphere contains single-mode particles having $n_{r}$ and effective radius $\left(r_{e}\right)$ given at the top. Comparison-space particles, having varying $n_{r}$, are defined above each panel; comparisons are made for three values of AOD and a range of geometries. The geographic placement of the plots is for illustration - over-water conditions are assumed everywhere, with the surface pressure prescribed as $1013.25 \mathrm{mb}$, and the surface wind speed set to $2.5 \mathrm{~m} / \mathrm{s}$.

\section{Parameter Space}

Simulated Atmosphere $r_{e}$ values $(\mu \mathrm{m}): 0.12,0.26,0.57,1.28,2.80$

Simulated Atmosphere $A O D$ values: $0.1,0.5,2.0$

Simulated Atmosphere $\boldsymbol{n}_{r}$ value: 1.45

Comparison Space $\boldsymbol{n}_{r}$ values: 1.35, 1.40, 1.50, 1.55 


\section{Input Atmospheric Particles: $\boldsymbol{n}_{r}=1.45, r_{e}=0.12 \mu m$}
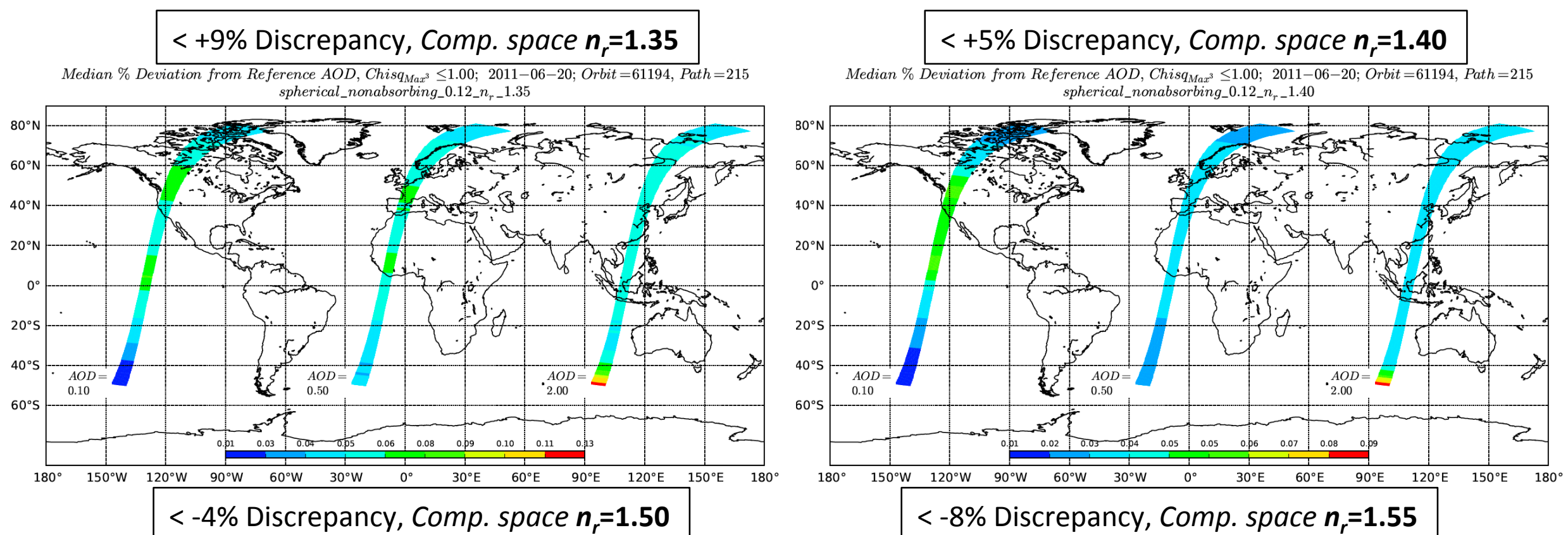

Median \% Deviation from Reference AOD, Chis Max $^{3} \leq 1.00 ; 2011-06-20 ;$ Orbit $=61194$, Path $=215$ spherical_nonabsorbing_0.12_ $n_{r-} 1.50$
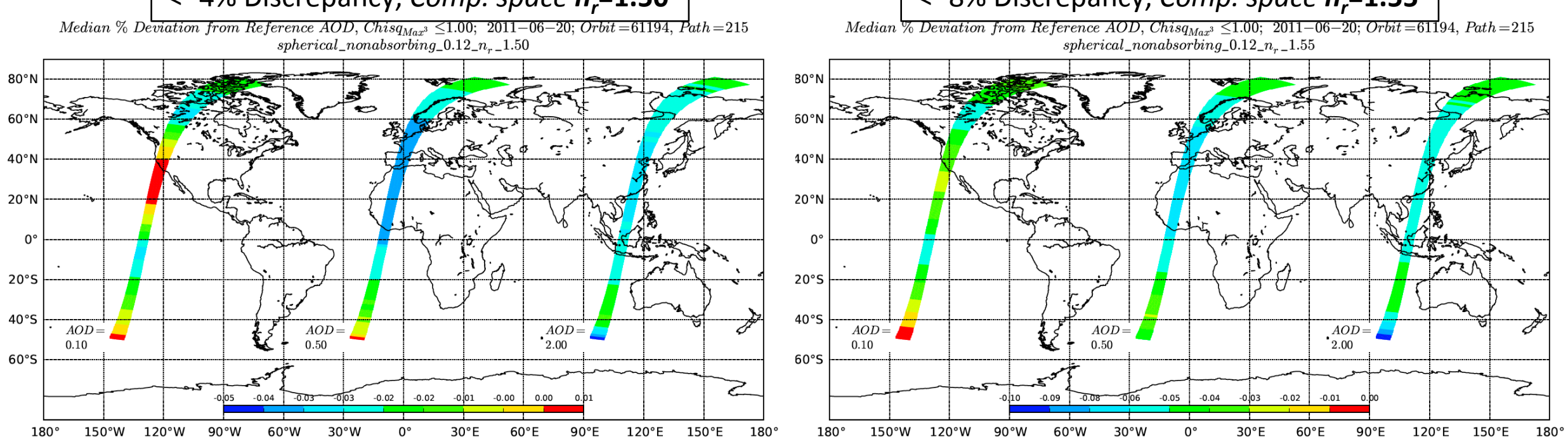

Figure S1 


\section{Input Atmospheric Particles: $n_{r}=1.45, r_{e}=0.26 \mu m$}
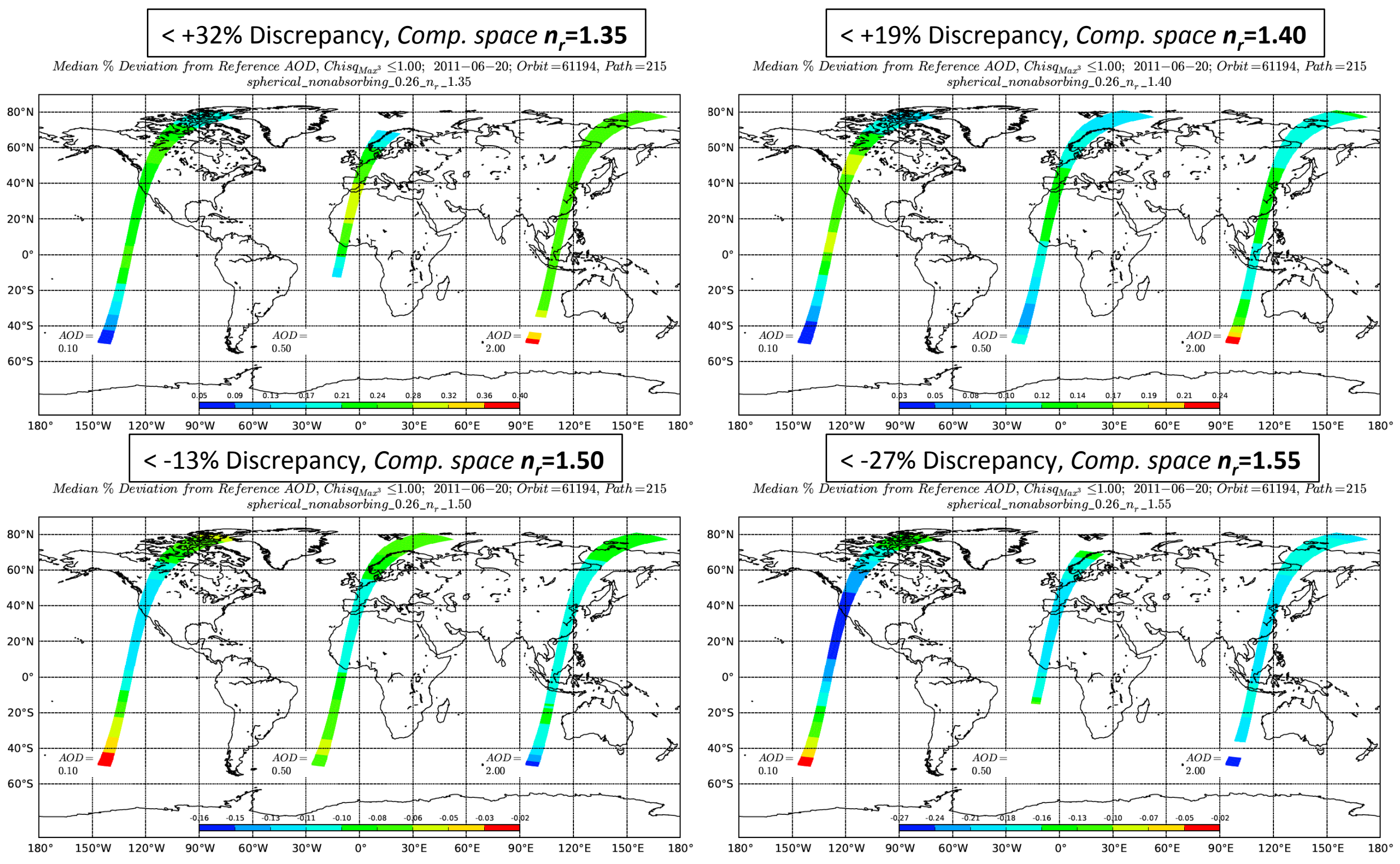

Figure S2 


\section{Input Atmospheric Particles: $n_{r}=1.45, r_{e}=0.57 \mu \mathrm{m}$}

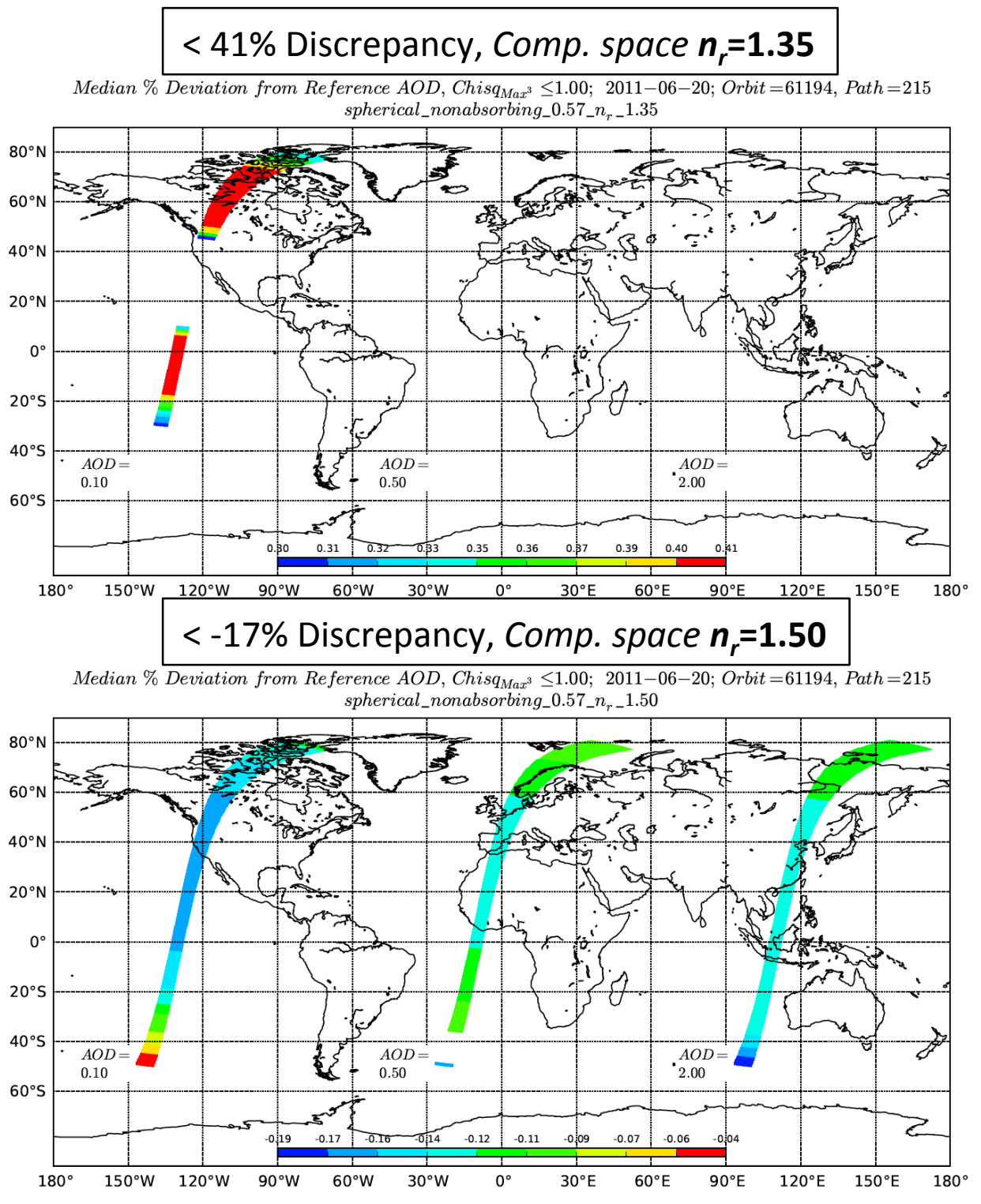

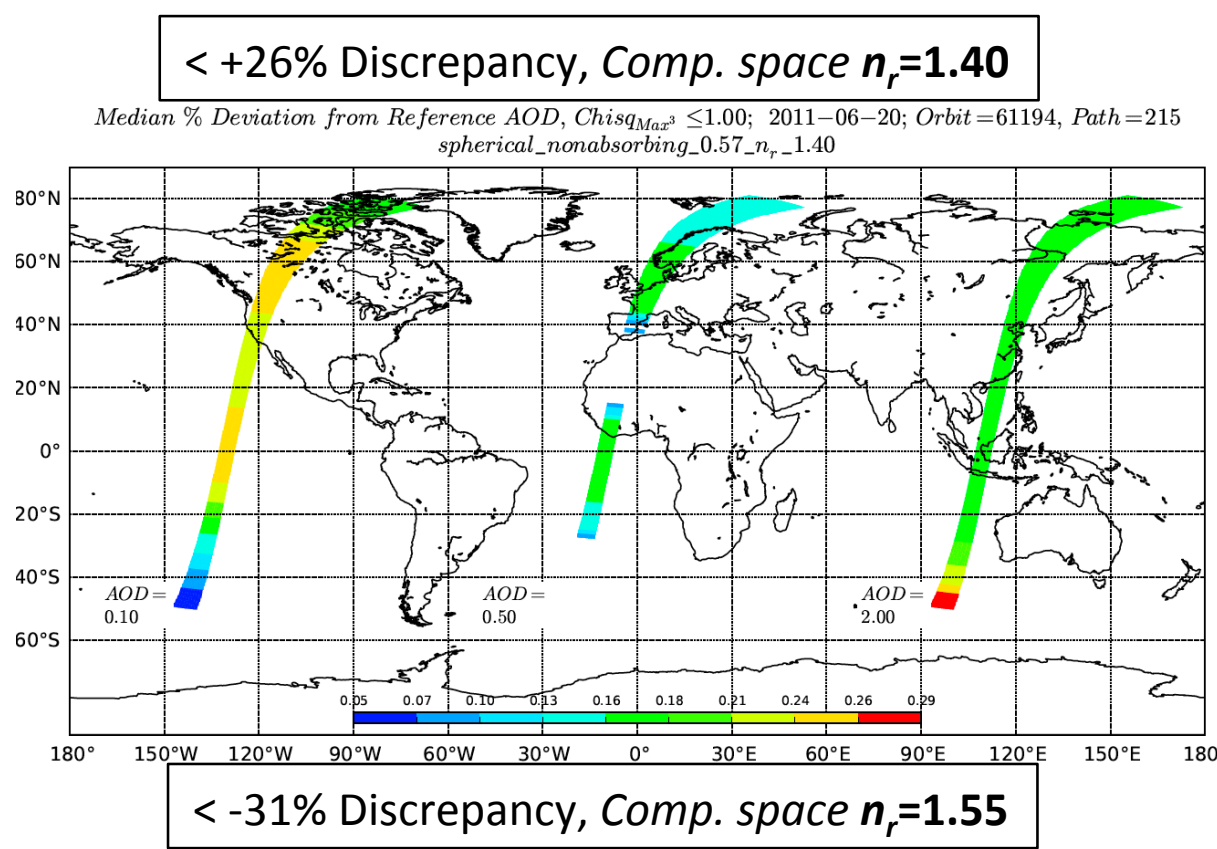

Median \% Deviation from Reference AOD, Chisq Max $^{3} \leq 1.00 ; 2011-06-20 ;$ Orbit $=61194$, Path $=215$ spherical_nonabsorbing_0.57_n $n_{r-1}-1.55$

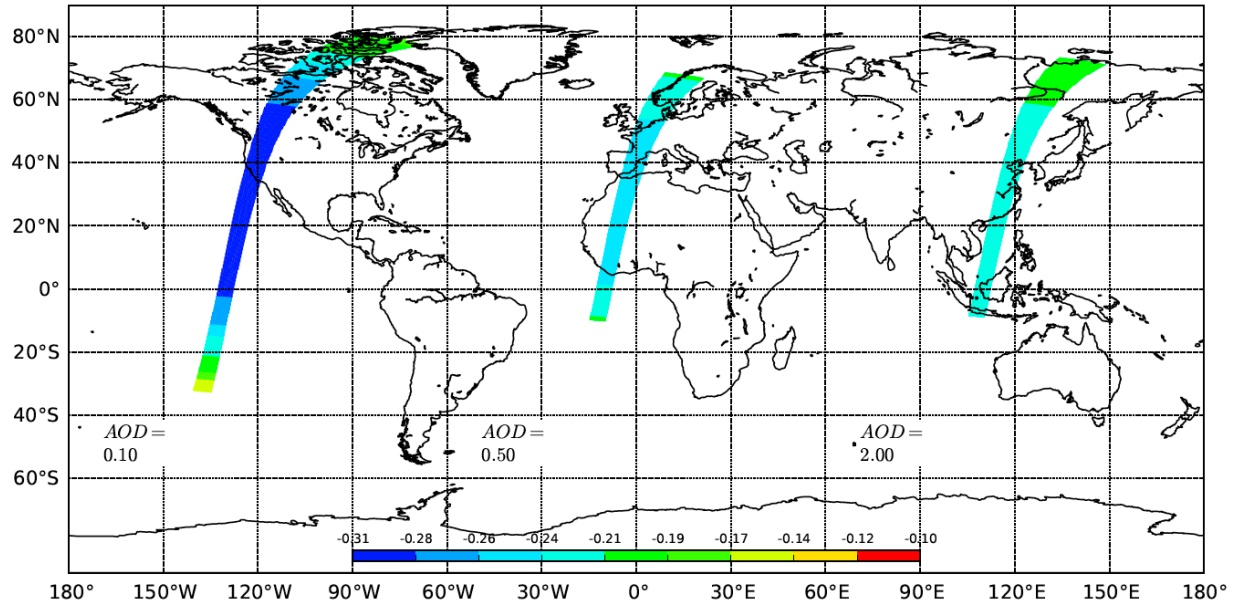

Figure S3 


\section{Input Atmospheric Particles: $n_{v}=1.45, r_{e}=1.28 \mu \mathrm{m}$}
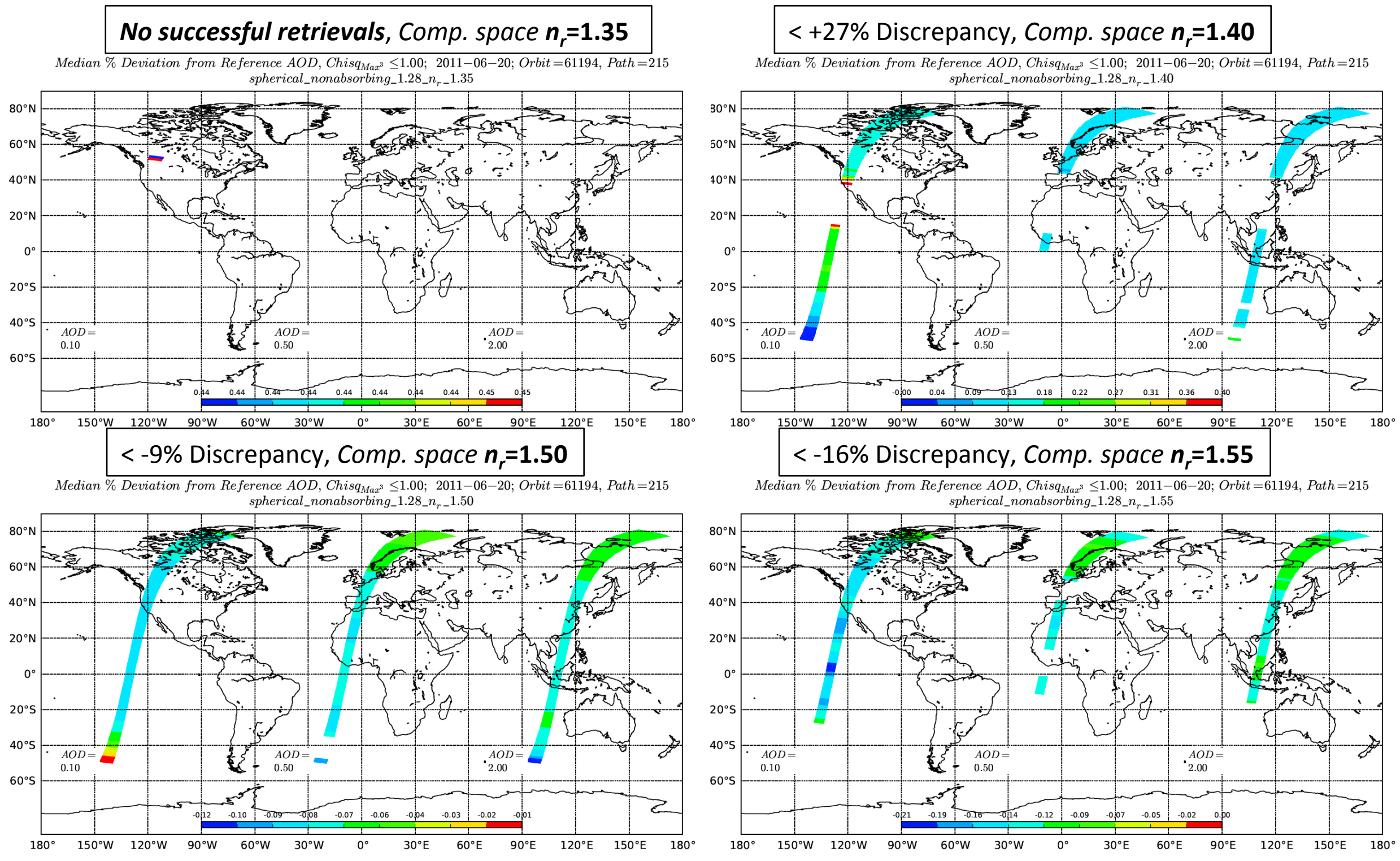

Median \% Deviation from Reference AOD, Chisq Max $^{3} \leq 1.00 ; 2011-06-20 ;$ Orbit $=61194$, Path $=215$ spherical_nonabsorbing_1.28_n $n_{r-} 1.55$

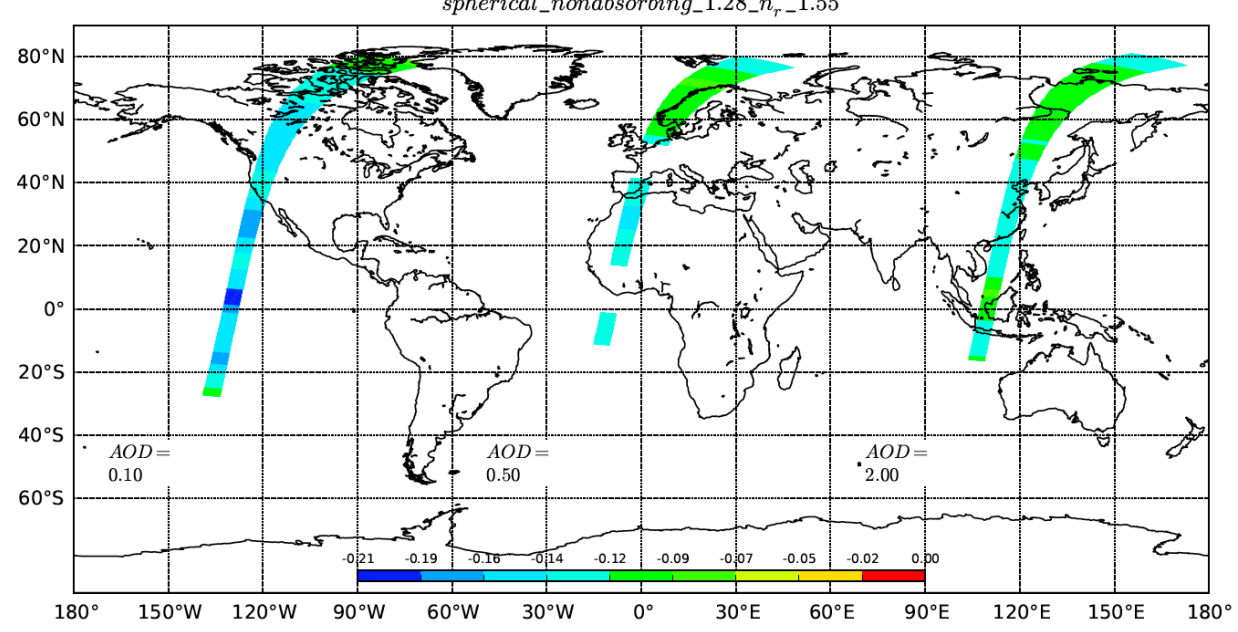

Figure S4 


\section{Input Atmospheric Particles: $n_{r}=1.45, r_{e}=2.80 \mu m$}

No successful retrievals, Comp. space $n_{r}=1.35$

\section{For very large particles,}

If $n_{r}$ deviates by 0.05 or more no successful retrievals are obtained (i.e., some retrieval sensitivity to $n_{r}$ )

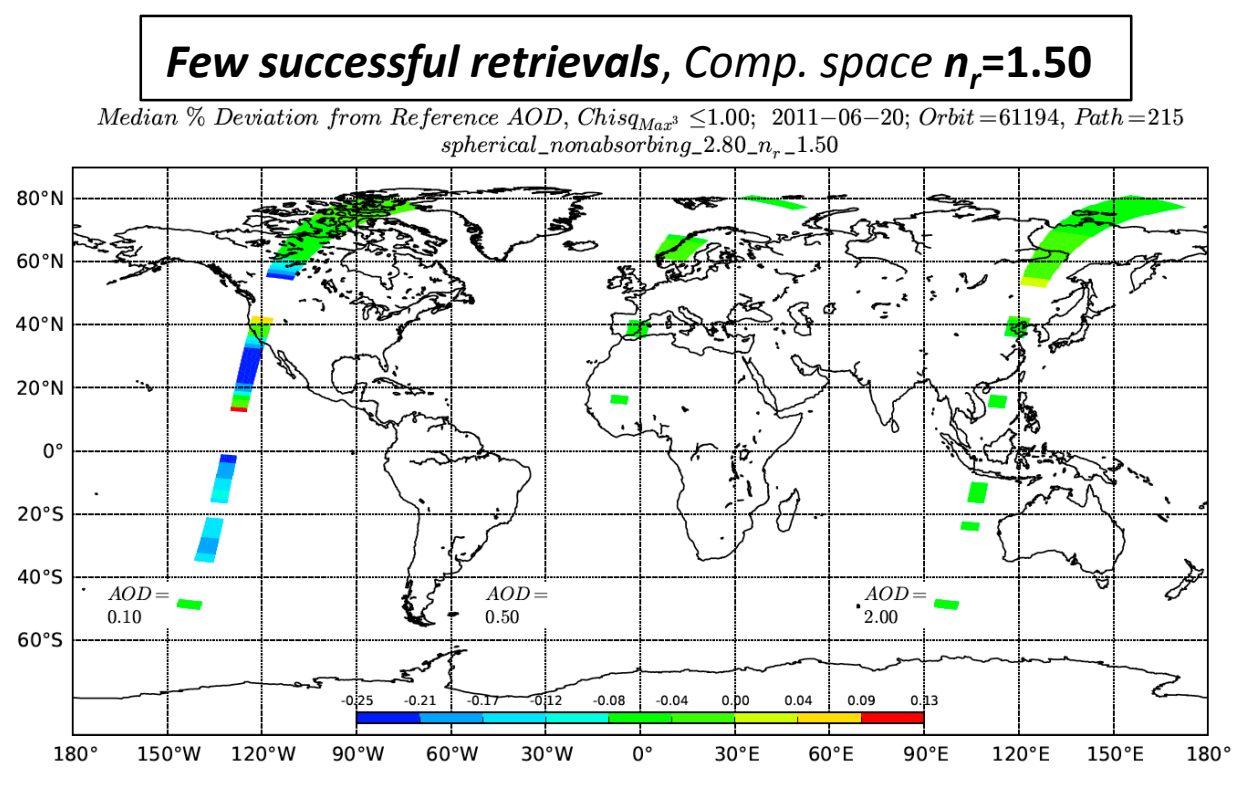

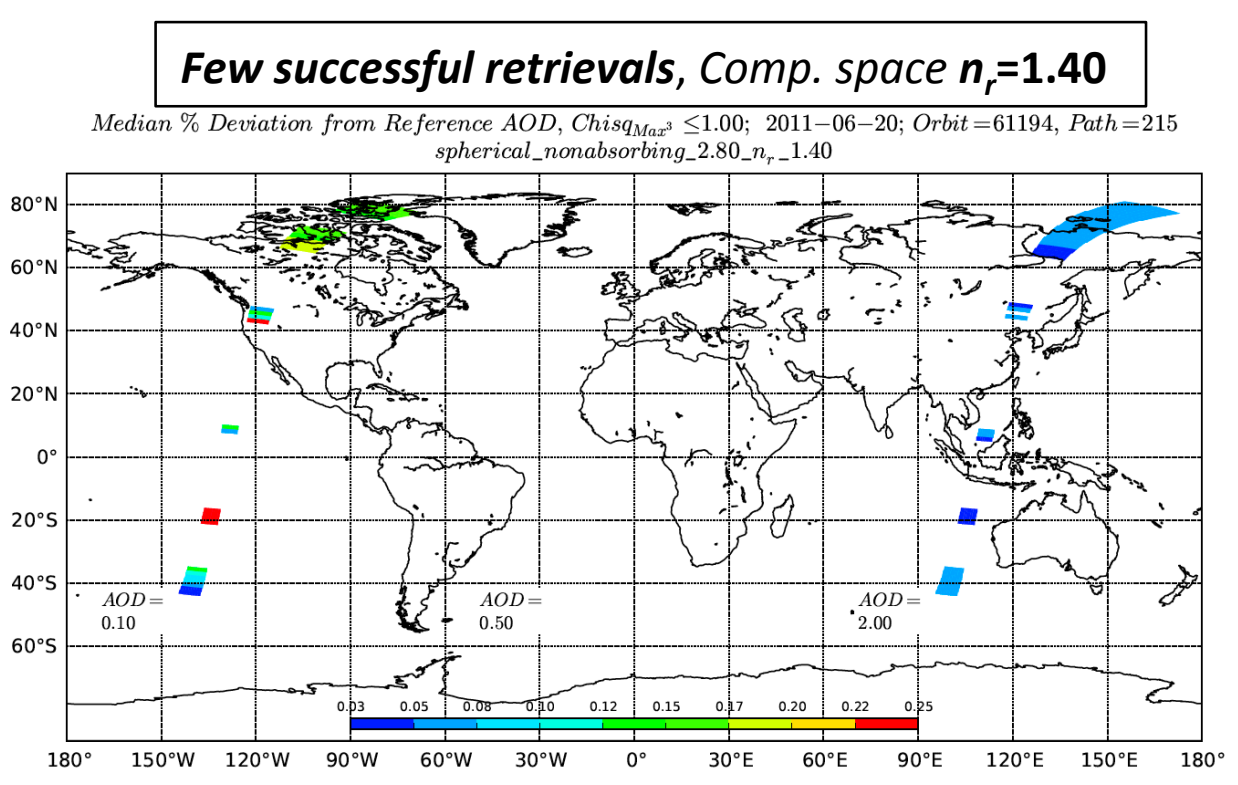

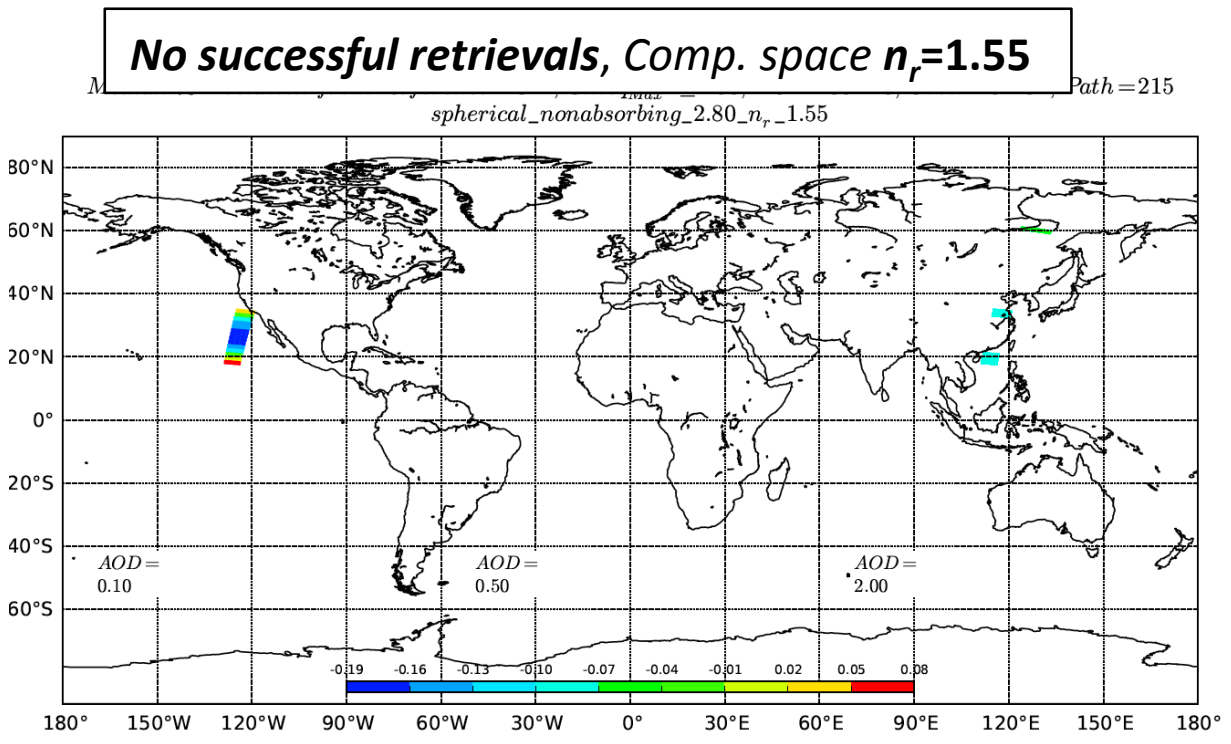

Figure S5 


\section{Real Refractive Index Sensitivity Study Conclusions}

\section{[Figures S1 - S5]}

- When $n_{r}$ is overestimated, AOD is systematically underestimated, and conversely

- Generally, retrieved AOD values still fall within 0.05 or 20\% AOD, except in extreme cases

- Smaller particles are affected less by errors in $n_{r}$

- Very large particles are so sensitive to changes in $n_{r}$ that mixtures might not pass the algorithm acceptance criteria if $n_{r}$ deviates too far ( 0.05$)$ from the correct value

- Medium particles $(0.26-0.57 \mu \mathrm{m})$ produce the largest AOD deviations for $\sim 0.1 n_{r}$ error, but are not sensitive enough to $n_{r}$ to retrieve the correct value. Summary:

- $\quad r_{e}=0.12 \mu \mathrm{m}: 5 \%-7.5 \%$ max. deviation for every 0.1 deviation from the correct $n_{r}$ [Figure S1]

- $\quad r_{e}=0.26 \mu \mathrm{m}: 20 \%-30 \%$ max. deviation for every 0.1 deviation from correct $n_{r}$ [Figure S2]

- $\quad r_{e}=0.57 \mu \mathrm{m}: 20 \%-40 \%$ max. deviation for every 0.1 deviation from correct $n_{r}$ [Figure S3]

- $\quad r_{e}=1.28 \mu \mathrm{m}: 15 \%-40 \%$ max. deviation for every 0.1 deviation from correct $n_{r}$ [Figure S4]

- $\quad r_{e}=2.80 \mu \mathrm{m}$ : variable max. deviation for every 0.1 deviation from correct $n_{r}$ [Figure S5]

- Distributions having larger effective radii tend to have biases that vary considerably depending on viewing/solar geometry

- Overall, the $0.57 \mu \mathrm{m}$ particle tends to perform the worst if $n_{r}$ is incorrect; the $0.26 \mu \mathrm{m}$ particle is a close second. (Mixtures might still pass, but the retrieved AOD discrepancy can be $>0.05 / 20 \%$.) 


\section{Linear Mixing (LM) \& Modified-Linear Mixing (MLM) Sensitivity Study}

This section expands on the AOD retrieval sensitivity analysis for Linear Mixing and Modified Linear Mixing, as illustrated in Figure 7 of the main paper. MLM is used to approximate the radiative effects of mixtures containing two or more optically distinct aerosol components, based on pre-run radiative transfer calculations for the components individually [Abdou et al., 1997]. This supplement considers bi-modal particle distributions covering a range of particle sizes and SSA values, comparing retrieved AOD results from LM and MLM with those derived from runs of the radiative transfer code using layer-effective phase function (Equs. 3 of the main text).

For each figure in this section, the simulated atmosphere and retrieval climatology contain the same bi-modal mixture of particles, specified in the figure annotation, and taken from the SA climatology [Kahn et al., 2010]. Comparisons are made between radiative transfer runs using layer-effective phase function and LM or MLM approximations, for five values of AOD and a range of geometries. (The geographic placement of the plots is for illustration - all retrievals are performed over simulated black surfaces.) Mixture numbers in the figures correspond to the climatology in the MISR V22 SA climatology.

[Note that $M L M$ reduces to $L M$ when all particles in the mixture are non-absorbing or have the same SSA.] 


\section{Impact of Linear Mixing; Globally, Non-Absorbing Aerosol}
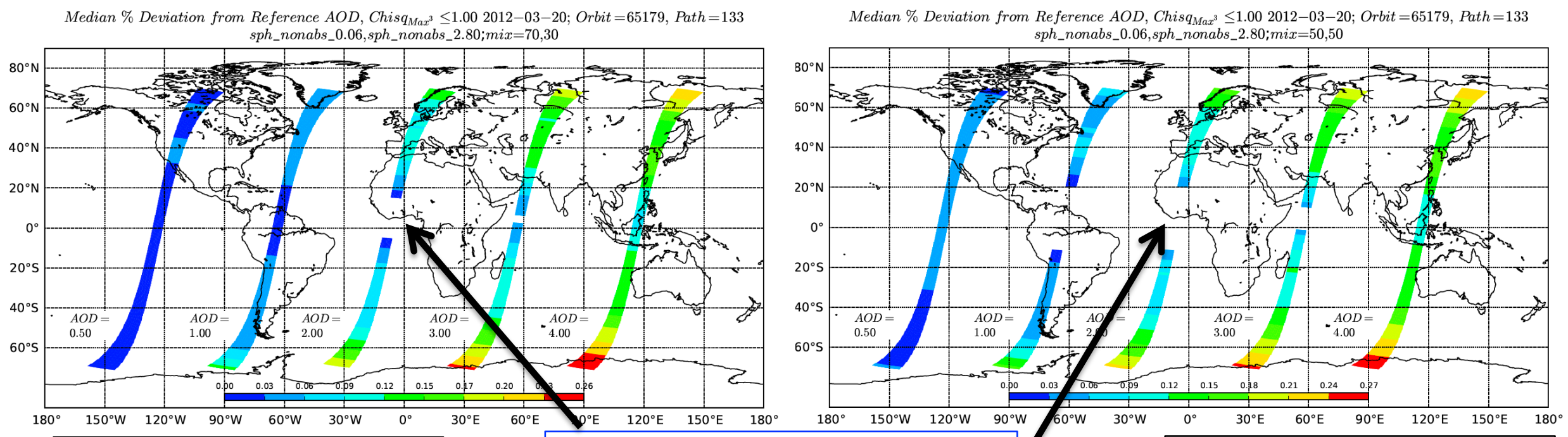

$<+17 \%$ Discrepancy, Mix \#5 70\% sph_nonabs_0.06 $30 \%$ sph_nonabs_2.80

$<+16 \%$ Discrepancy, Mix \#9 30\% sph_nonabs_0.06 70\% sph_nonabs_2.80
Note: At low lat, moderate AOD $\rightarrow$ No mixtures pass when LM is used

Median \% Deviation from Reference AOD, Chis $_{M_{a x}{ }^{3}} \leq 1.00$ 2012-03-20; Orbit $=65179$, Path $=133$ sph_nonabs_0.06,sph_nonabs_2.80;mix $=30,7$

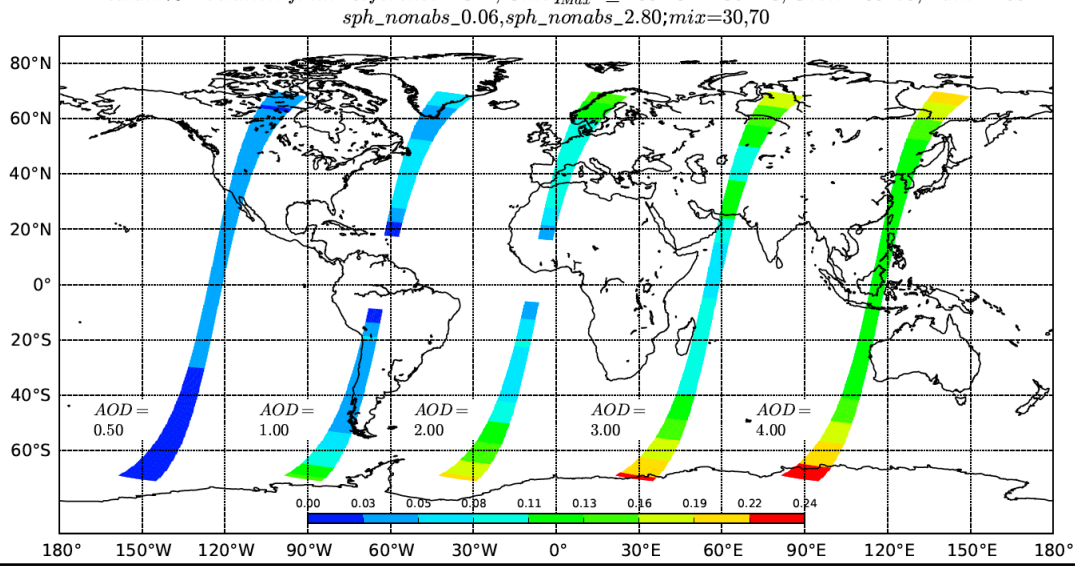

$<+18 \%$ Discrepancy, Mix \#7 $50 \%$ sph_nonabs_0.06 $50 \%$ sph_nonabs_2.80

$0.06+2.80 \mu \mathrm{m}$ $r_{e}$ particles

- Linear mixing overestimates AOD with spherical non-absorbing particles

- A larger particle size difference $\rightarrow$ larger AOD overestimate 


\section{Impact of Linear Mixing; Globally, Non-Absorbing Aerosol}

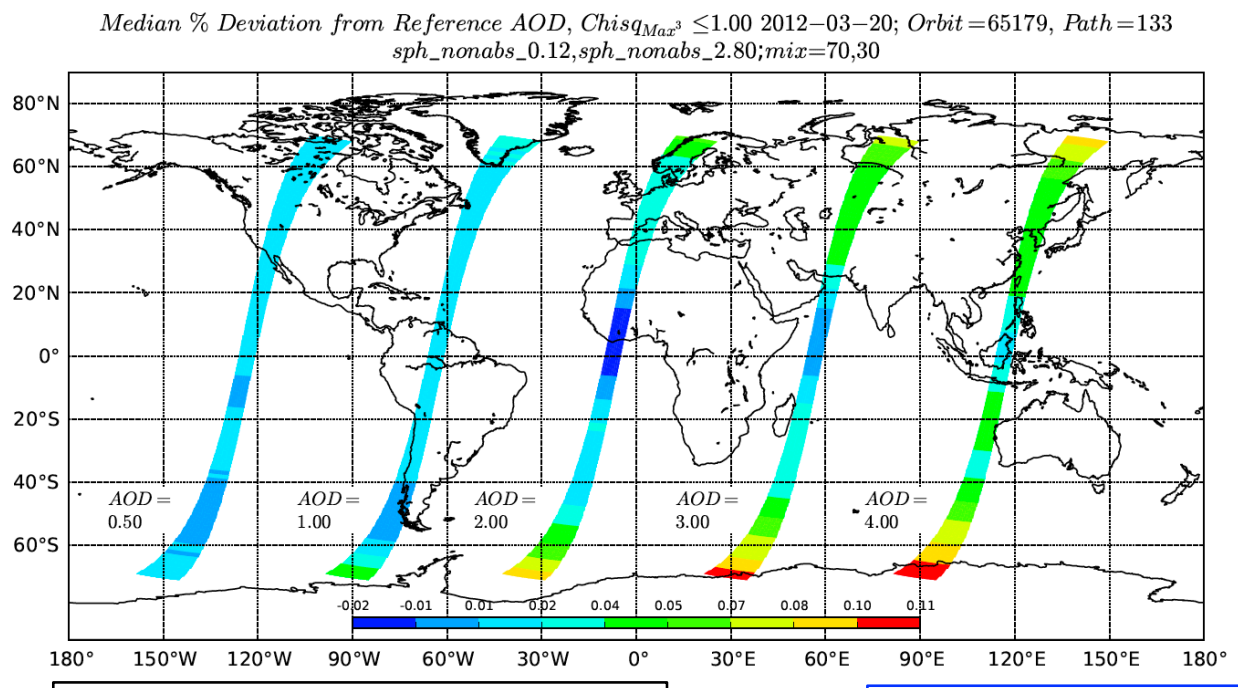

$<+7 \%$ Discrepancy, Mix \#15 70\% sph_nonabs_0.12 $30 \%$ sph_nonabs_2.80

$<+7 \%$ Discrepancy, Mix \#19 $30 \%$ sph_nonabs_0.12 70\% sph_nonabs_2.80
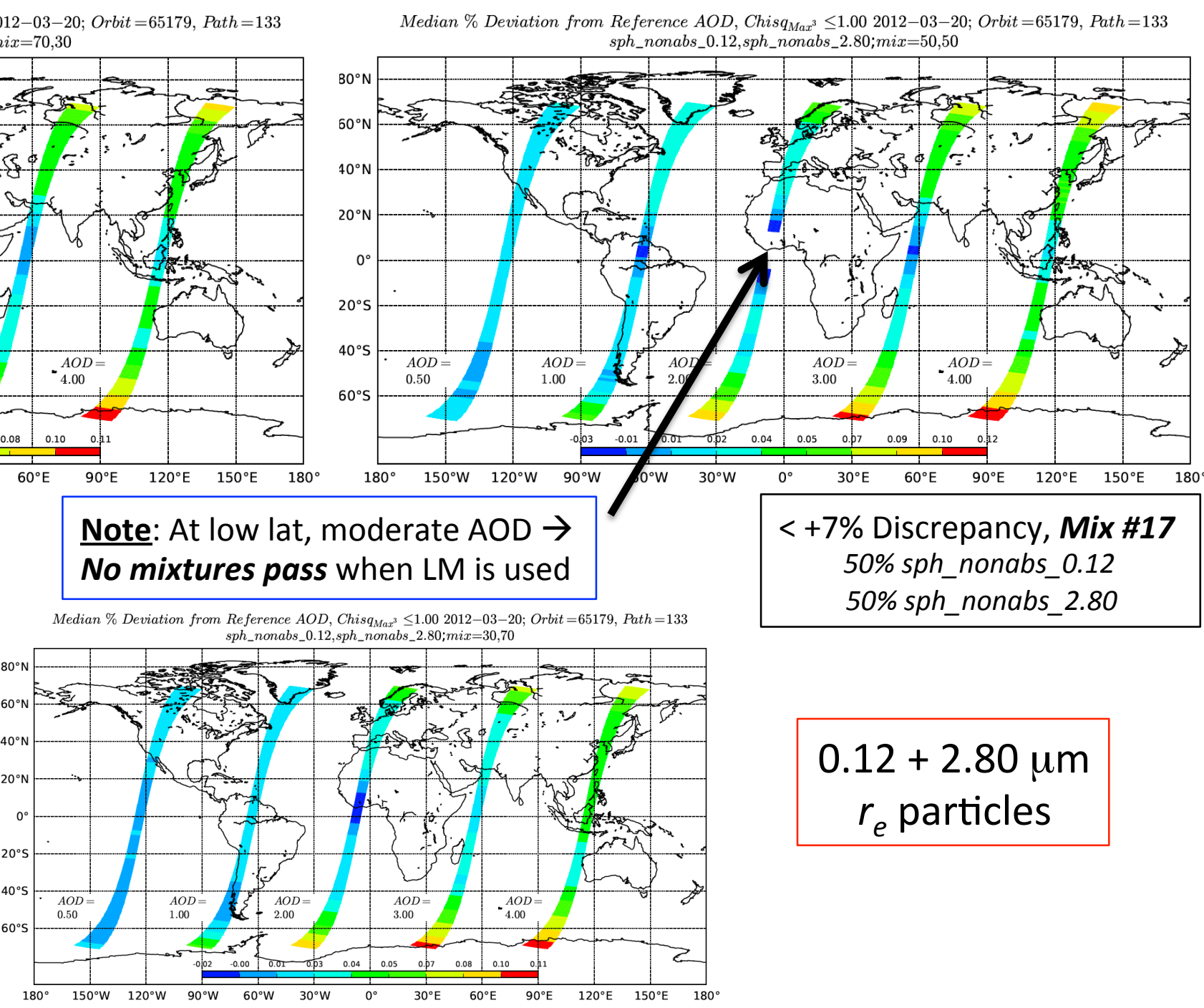

Note: At low lat, moderate AOD $\rightarrow$ No mixtures pass when LM is used $50 \%$ sph_nonabs_0.12 $50 \%$ sph_nonabs_2.80

$0.12+2.80 \mu \mathrm{m}$ $r_{e}$ particles

- Linear mixing overestimates AOD with spherical non-absorbing particles

- A larger particle size difference $\rightarrow$ larger AOD overestimate 


\section{Impact of Linear Mixing; Globally, Non-Absorbing Aerosol}

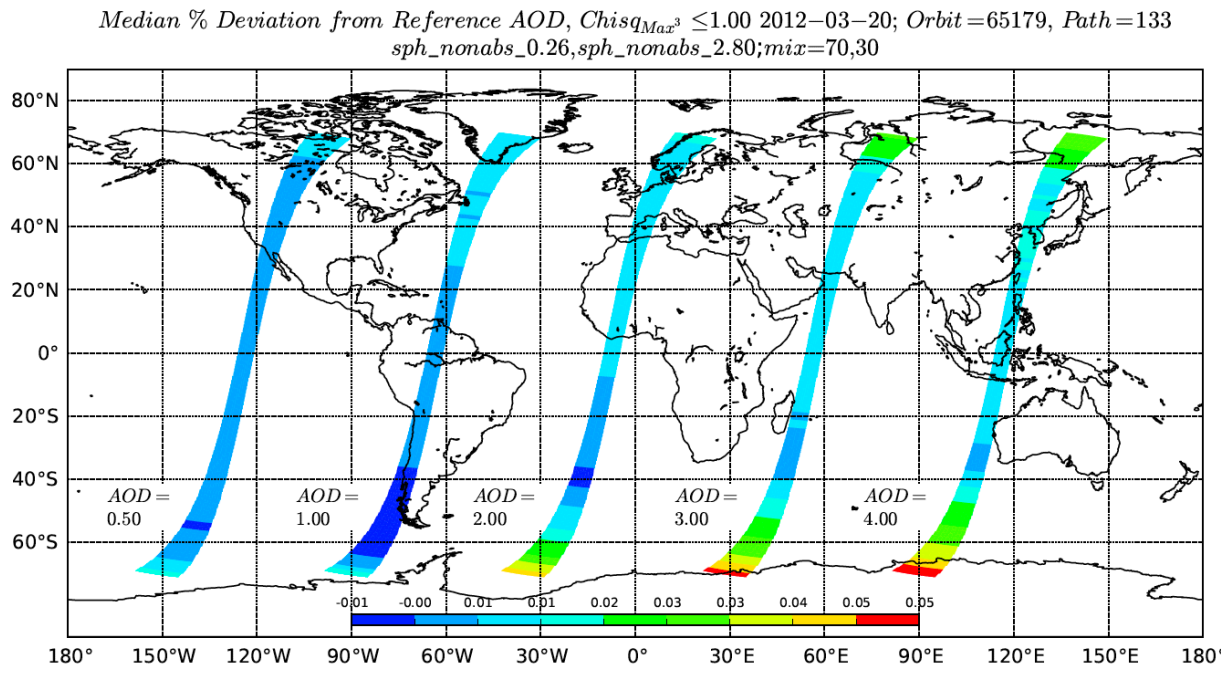

$<+2 \%$ Discrepancy, Mix \#25

$70 \%$ sph_nonabs_0.26

$30 \%$ sph_nonabs_2.80

< +3\% Discrepancy, Mix \#29

$30 \%$ sph_nonabs_0.26

$70 \%$ sph_nonabs_2.80
Median \% Deviation from Reference AOD, Chisq $q_{\mathrm{N}^{3}} \leq 1.002012-03-20$; Orbit $=65179$, Path $=133$

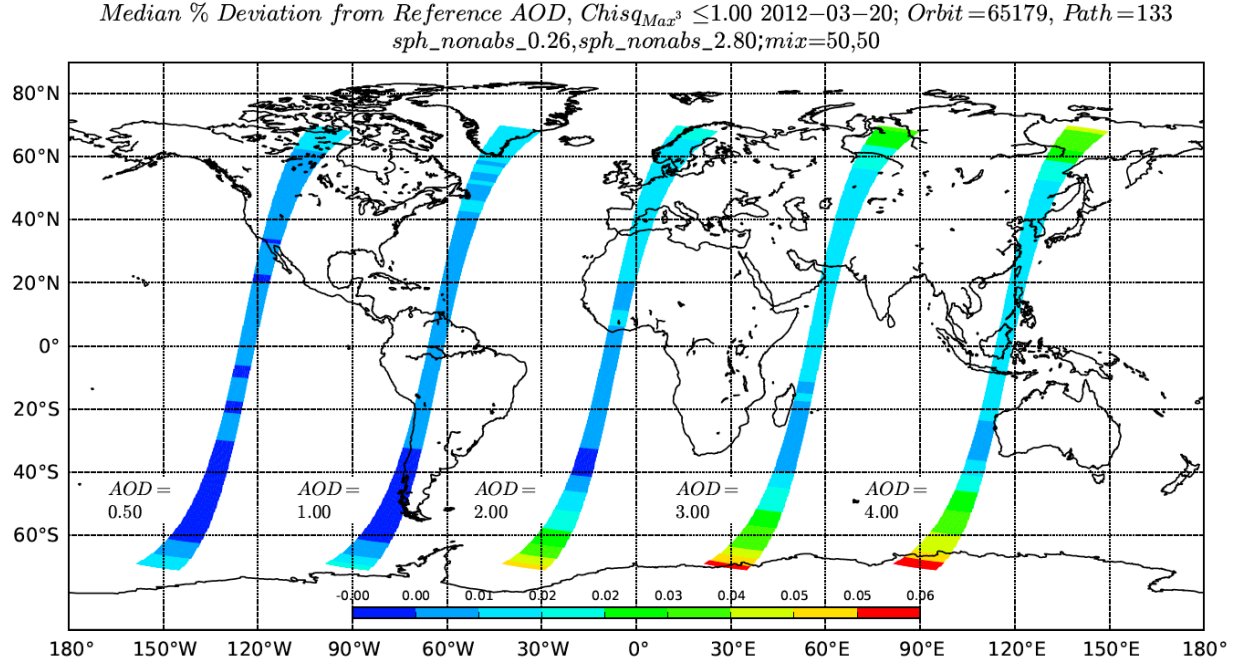

$<+2 \%$ Discrepancy, Mix \#27 $50 \%$ sph_nonabs_0.26 $50 \%$ sph_nonabs_2.80

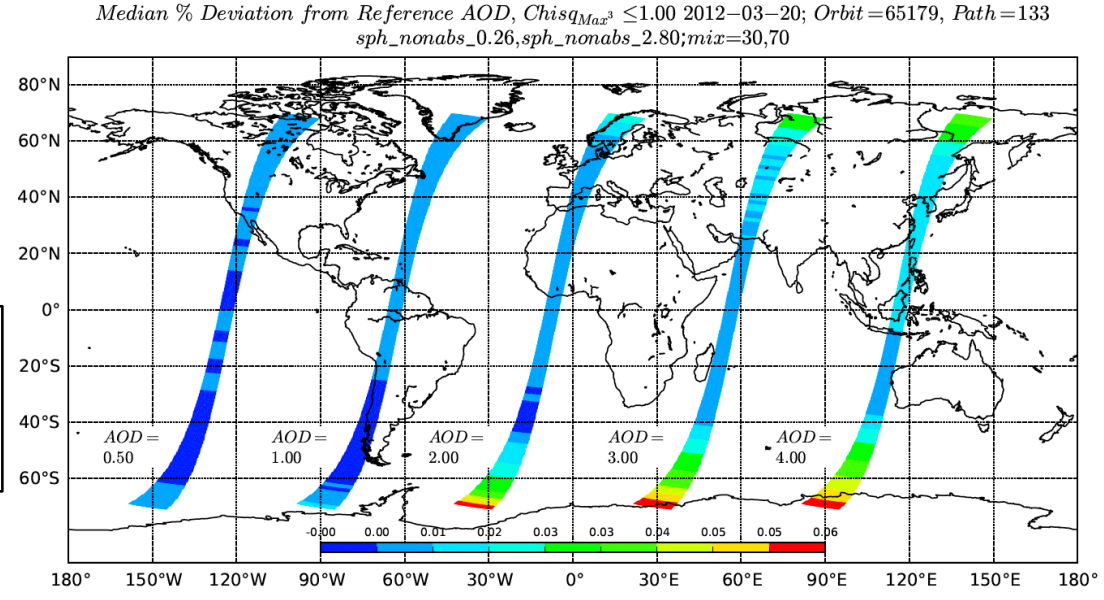

$0.26+2.80 \mu \mathrm{m}$ $r_{e}$ particles

- Linear mixing overestimates AOD with spherical non-absorbing particles

- A larger particle size difference $\rightarrow$ larger AOD overestimate 


\section{Impact of Linear Mixing; Globally, Non-Absorbing Aerosol}
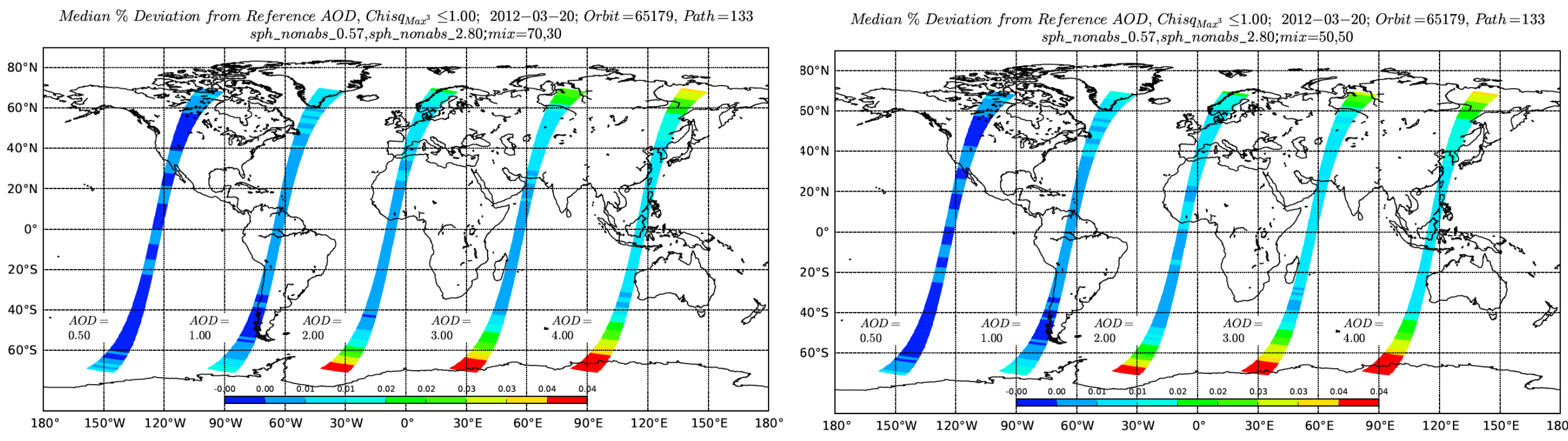

$<+2 \%$ Discrepancy 70\% sph_nonabs_0.57 $30 \%$ sph_nonabs_2.80

$<+2 \%$ Discrepancy $30 \%$ sph_nonabs_0.57 70\% sph_nonabs_2.80

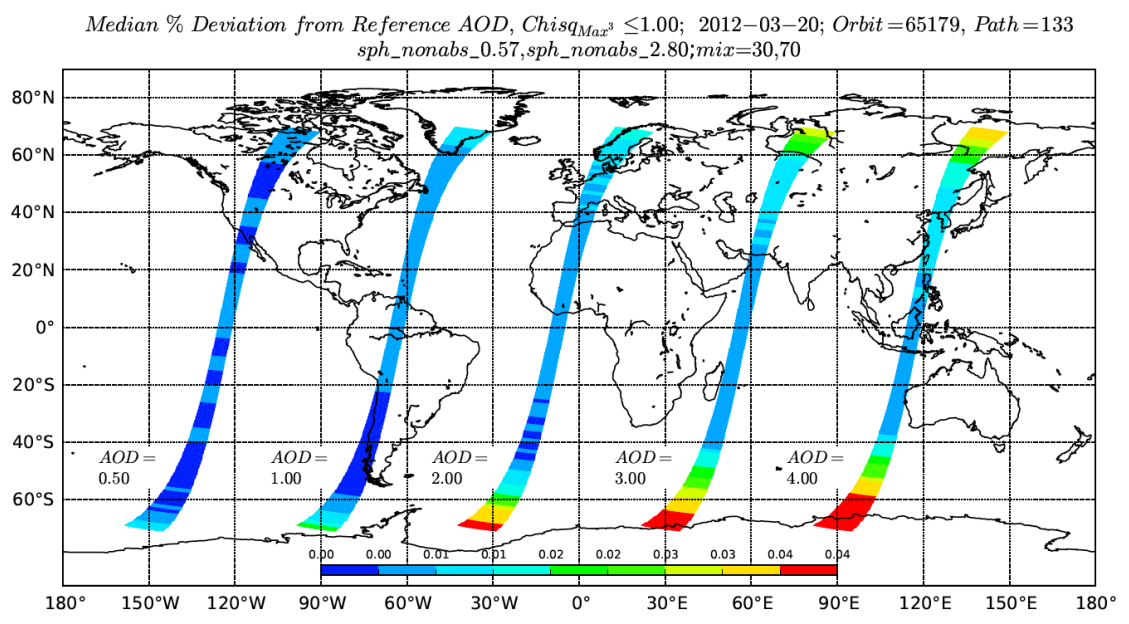

$<+2 \%$ Discrepancy 50\% sph_nonabs_0.57 50\% sph_nonabs_2.80

\section{$0.57+2.80 \mu \mathrm{m}$} $r_{e}$ particles

- Linear mixing overestimates AOD with spherical non-absorbing particles

- A larger particle size difference $\rightarrow$ larger AOD overestimate 


\section{Impact of Linear Mixing; Globally, Non-Absorbing Aerosol}

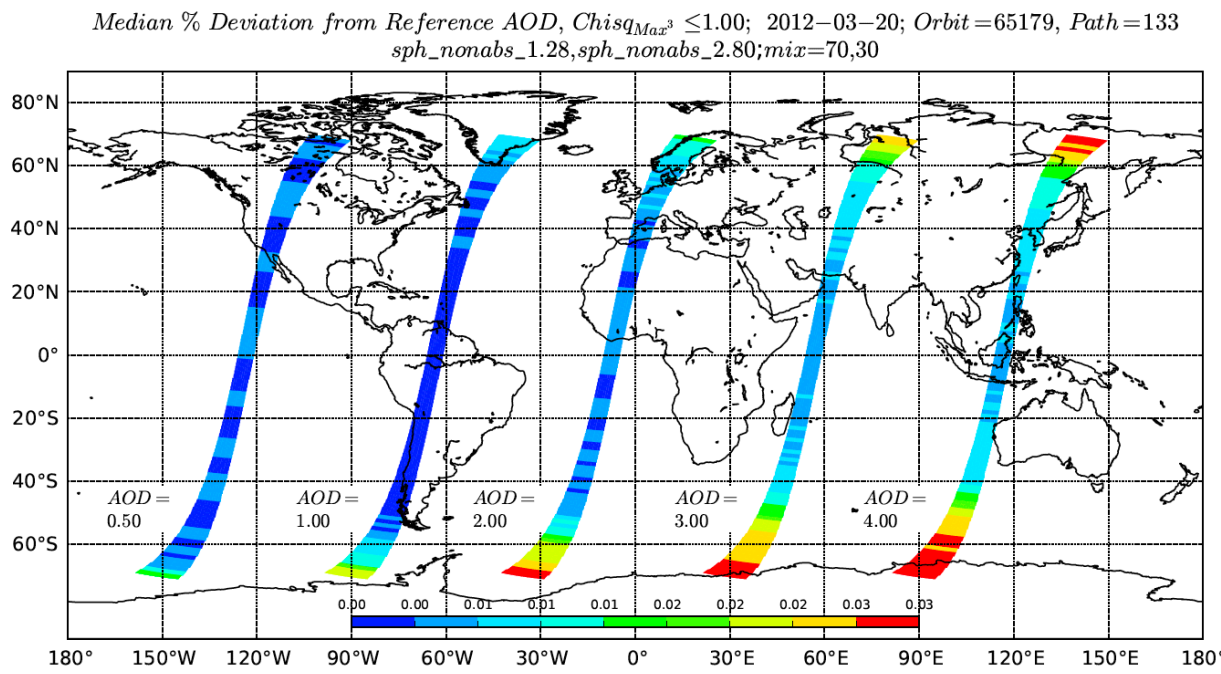

$<+1 \%$ Discrepancy $70 \%$ sph_nonabs_1.28 $30 \%$ sph_nonabs_2.80

$<+1 \%$ Discrepancy $30 \%$ sph_nonabs_1.28 $70 \%$ sph_nonabs_2.80
Median \% Deviation from Reference AOD, Chisqu $\leq 1.00 ; 2012-03-20 ;$ Orbit $=65179$, Path $=133$

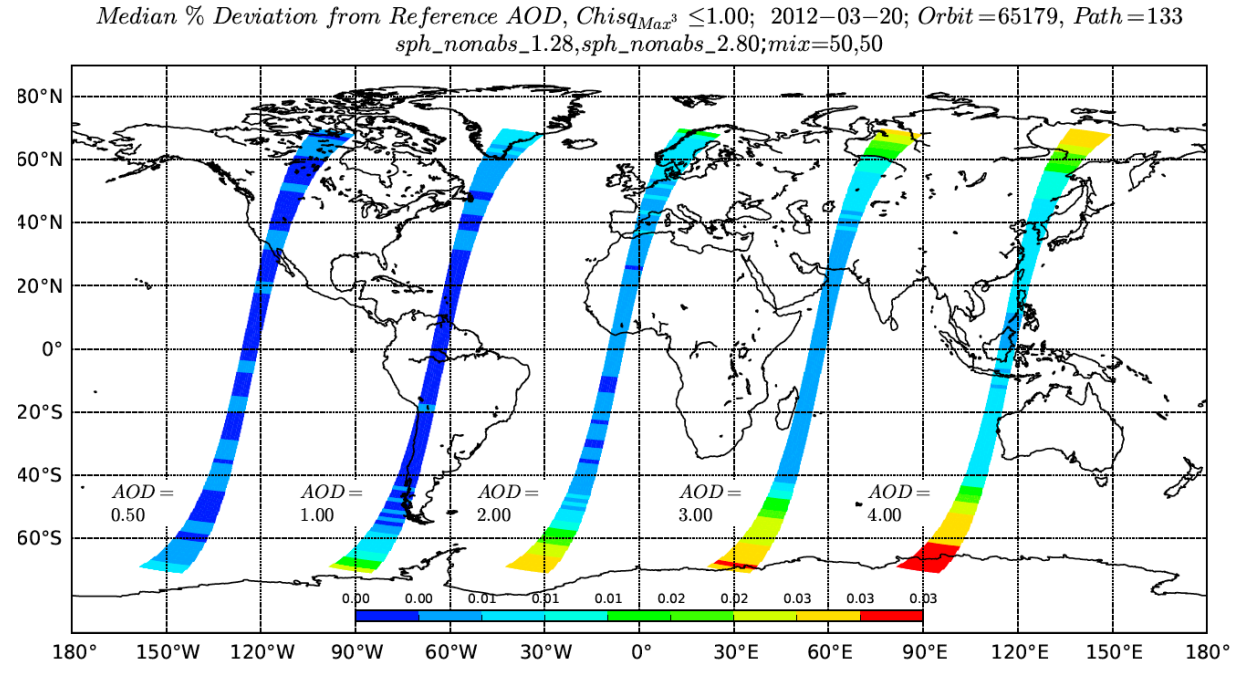

Median \% Deviation from Reference AOD, Chisq 3 <1.00; 2012-03-20; Orbit $=65179$, Path $=133$ sph_nonabs_1.28,sph_nonabs_2 $280 ;$ mix $=30,70$

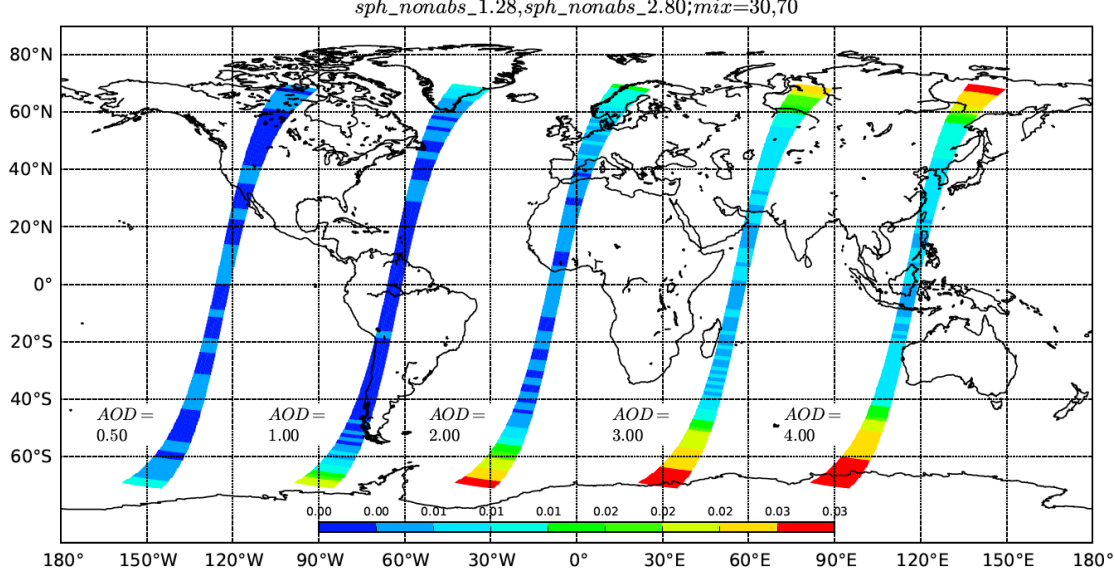

$1.28+2.80 \mu \mathrm{m}$

$r_{e}$ particles

- Linear mixing overestimates AOD with spherical non-absorbing particles

- A larger particle size difference $\rightarrow$ larger AOD overestimate 


\section{Impact of MLM; Globally, Absorbing Mixtures}
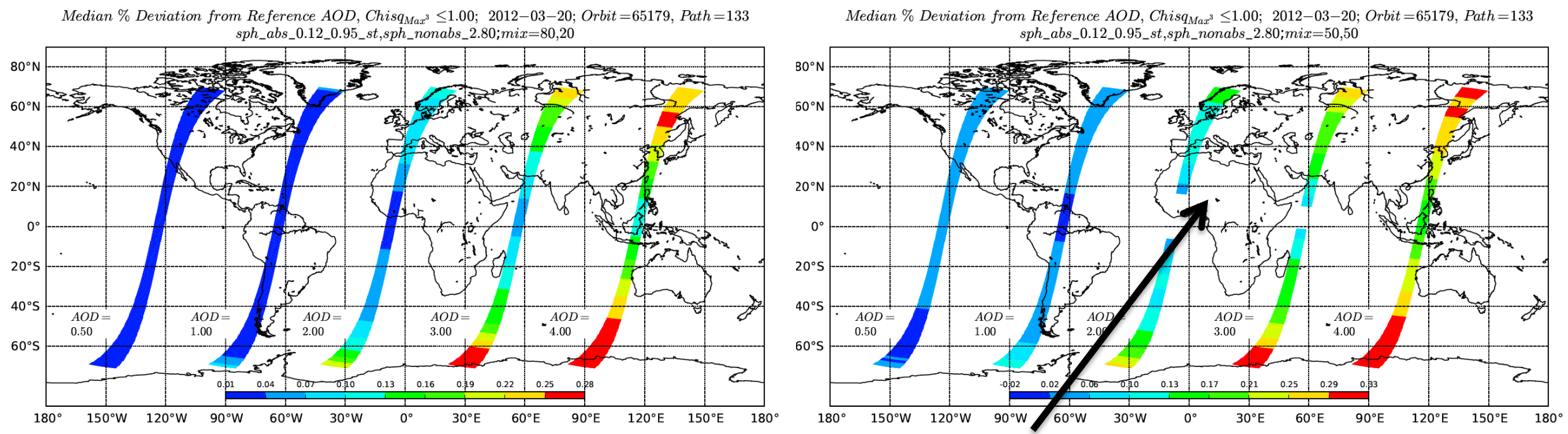

$<+25 \%$ Discrepancy $80 \%$ sph_abs_0.12_SSA-0.95 20\% sph_nonabs_2.80

$<+13 \%$ Discrepancy 20\% sph_abs_0.12_SSA-0.95 $80 \%$ sph_nonabs_2.80
Note: At low lat \& moderate AOD $\rightarrow$ No mixtures pass when MLM is used

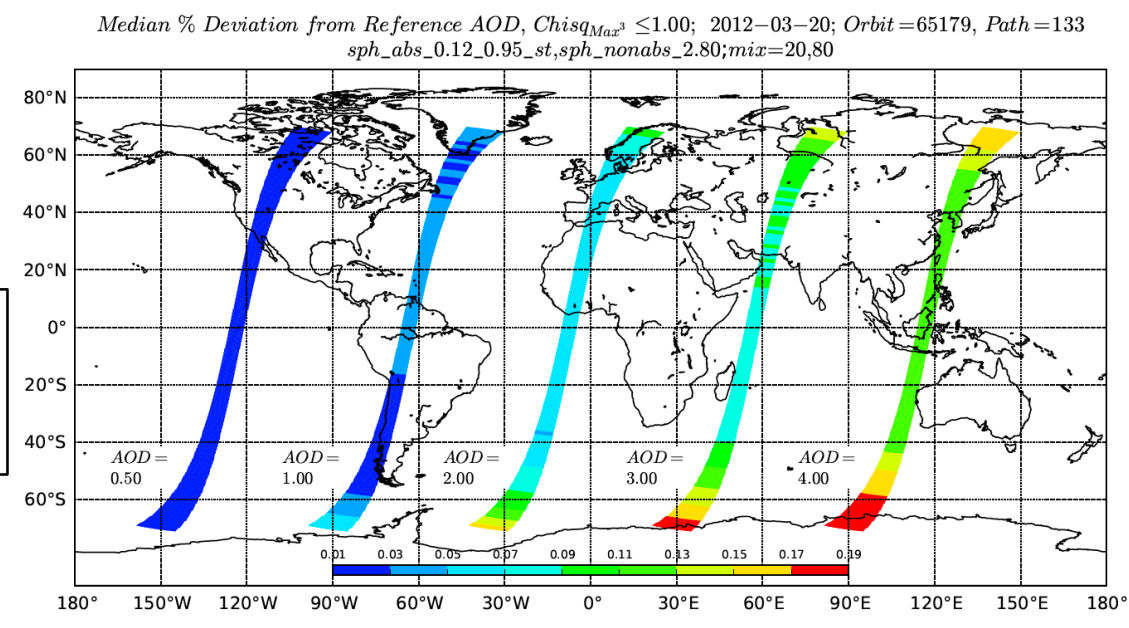

$<+29 \%$ Discrepancy 50\% sph_abs_0.12_SSA-0.95 $50 \%$ sph_nonabs_2.80

$$
\begin{gathered}
0.12 \text { SSA_0.95 } \\
+2.80 \mu \mathrm{m} \\
r_{e} \text { particles }
\end{gathered}
$$

- MLM overestimates AOD with spherical non-absorbing/absorbing particle mixtures 


\section{Impact of MLM; Globally, Absorbing Mixtures}

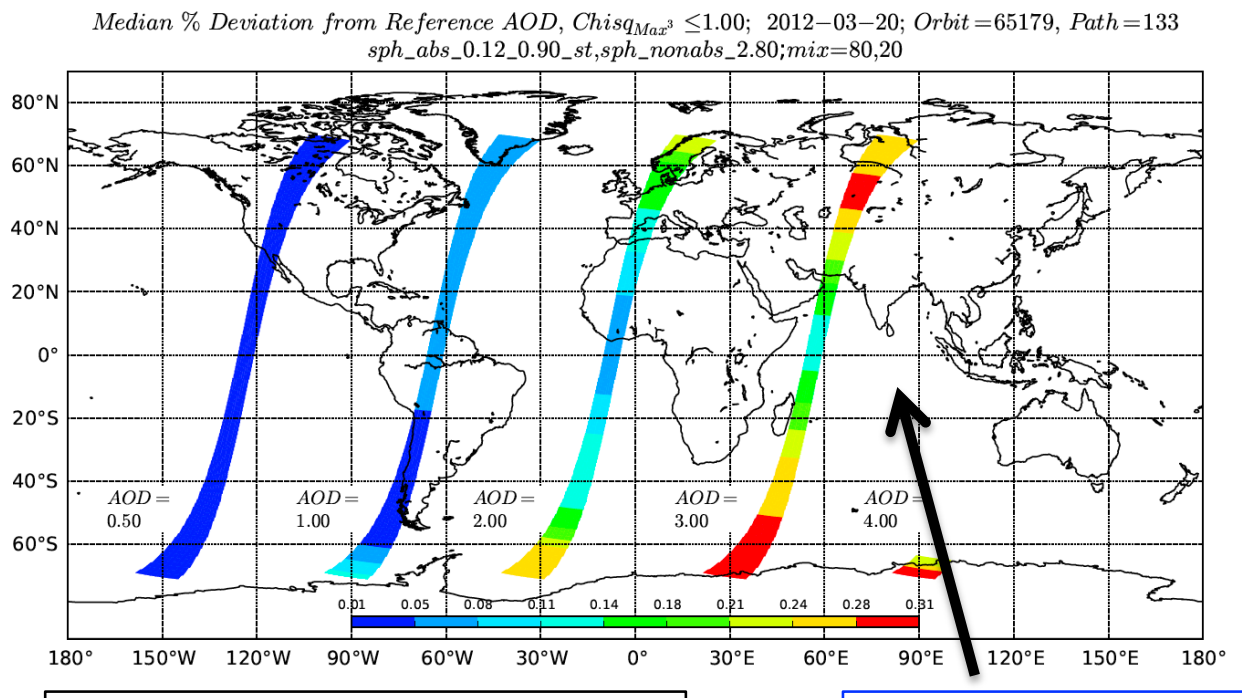

$<+28 \%$ Discrepancy, Mix \#34 $80 \%$ sph_abs_0.12_SSA-0.90 $20 \%$ sph_nonabs_2.80

< +17\% Discrepancy, Mix \#40 20\% sph_abs_0.12_SSA-0.90 $80 \%$ sph_nonabs_2.80

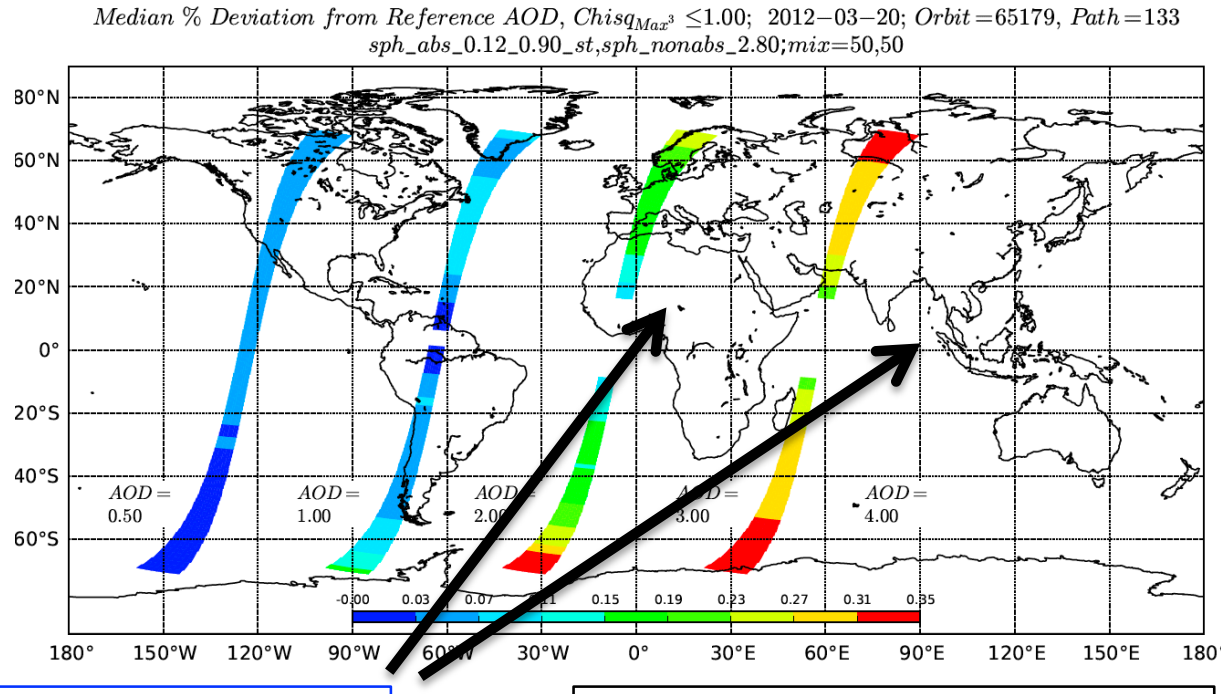

Note: At low lat \& high AOD $\rightarrow$ No mixtures pass when MLM is used

Median \% Deviation from Reference AOD, Chisq ${ }_{M_{a x}{ }^{3}} \leq 1.00 ; 2012-03-20 ;$ Orbit $=65179$, Path $=133$ sph_abs_0.12_0.90_st,sph_nonabs_2.80;mix =20,80

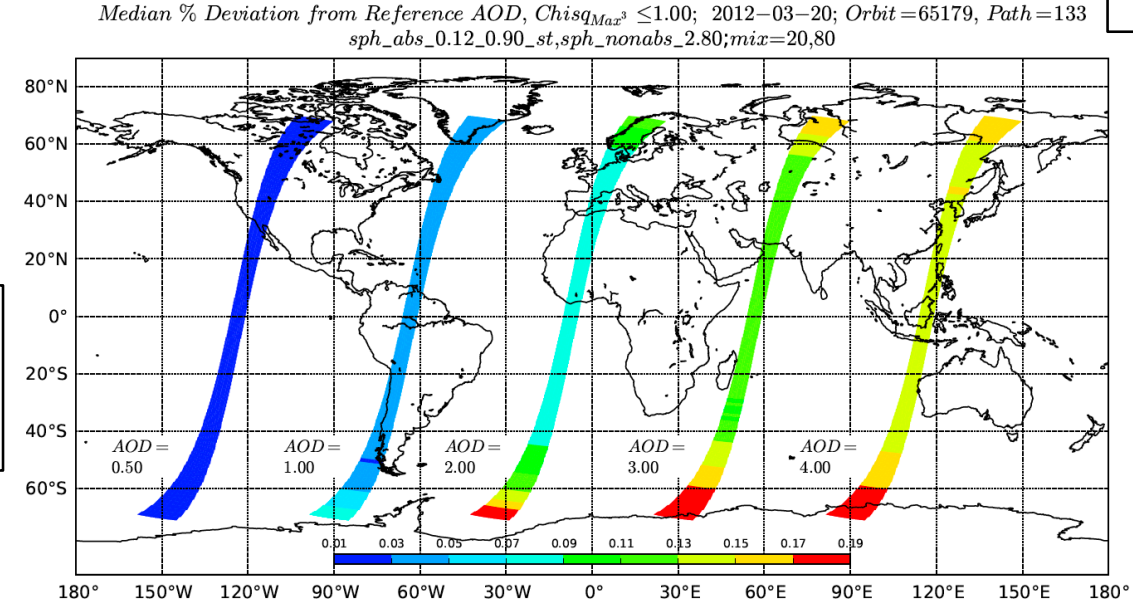

$<+31 \%$ Discrepancy, Mix \#37 $50 \%$ sph_abs_0.12_SSA-0.90 $50 \%$ sph_nonabs_2.80

$$
\begin{gathered}
0.12 \text { SSA_0.90 } \\
+2.80 \mu \mathrm{m} \\
r_{e} \text { particles }
\end{gathered}
$$

- MLM overestimates AOD with spherical non-absorbing/absorbing particle mixtures 


\section{Impact of MLM; Globally, Absorbing Mixtures}

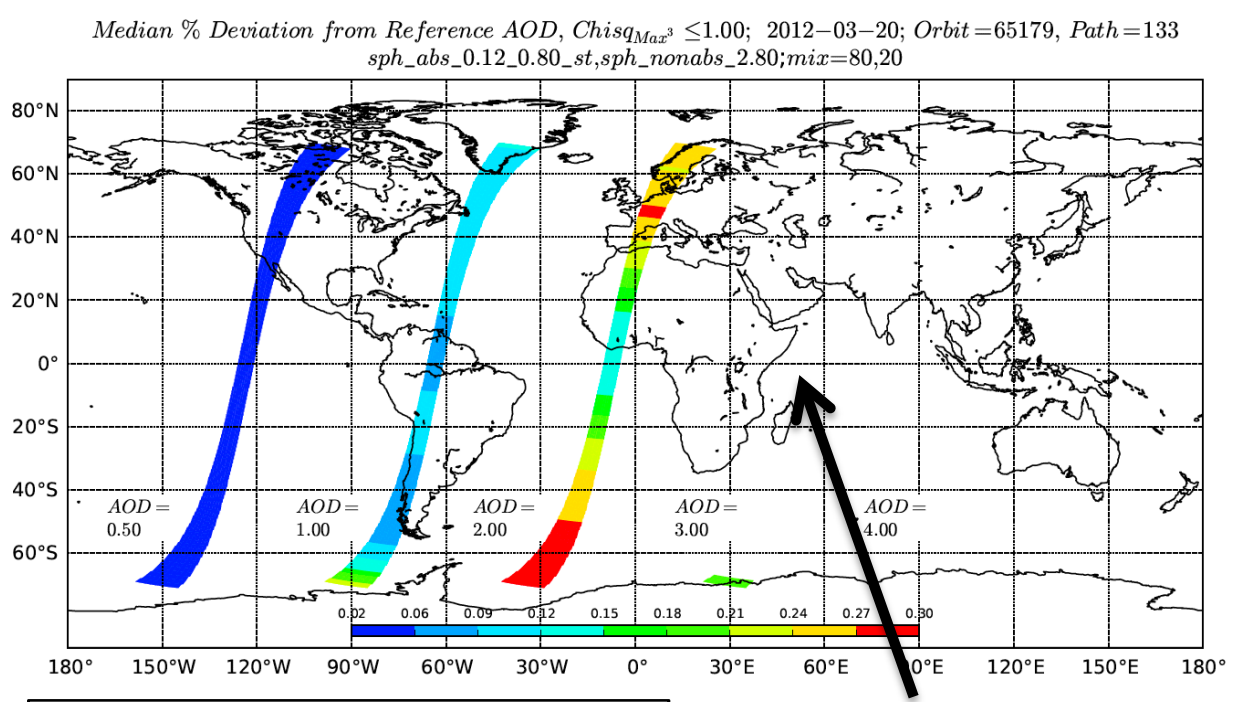

$<+27 \%$ Discrepancy, Mix \#44 $80 \%$ sph_abs_0.12_SSA-0.80 $20 \%$ sph_nonabs_2.80

\section{$<+12 \%$ Discrepancy, Mix \#50 20\% sph_abs_0.12_SSA-0.80 $80 \%$ sph_nonabs_2.80}

Median \% Deviation from Reference AOD, Chisq Max $^{3} \leq 1.00 ; 2012-03-20$; Orbit $=65179$, Path $=133$ $s p h \_a b s \_0.12 \_0.80 \_s t, s p h \_n o n a b s \_2.80 ; m i x=50,50$

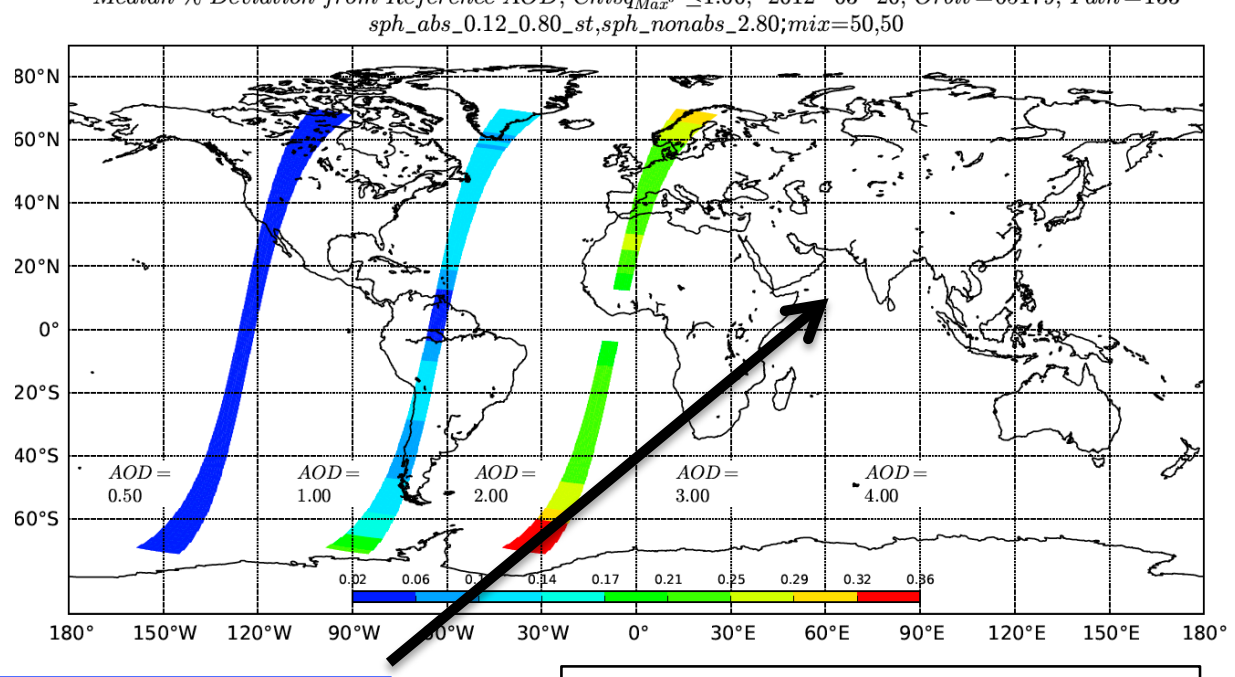

$<+29 \%$ Discrepancy, Mix \#47 $50 \%$ sph_abs_0.12_SSA-0.80 $50 \%$ sph nonabs 2.80

\section{No mixtures pass when MLM is used}

Median \% Deviation from Reference AOD, Chis $2 \leq 1.00 ; 2012-03-20 ;$ Orbit $=65179$, Path $=133$ sph_abs_0.12_0.80_st,sph_nonabs_2.80;mix =20,80

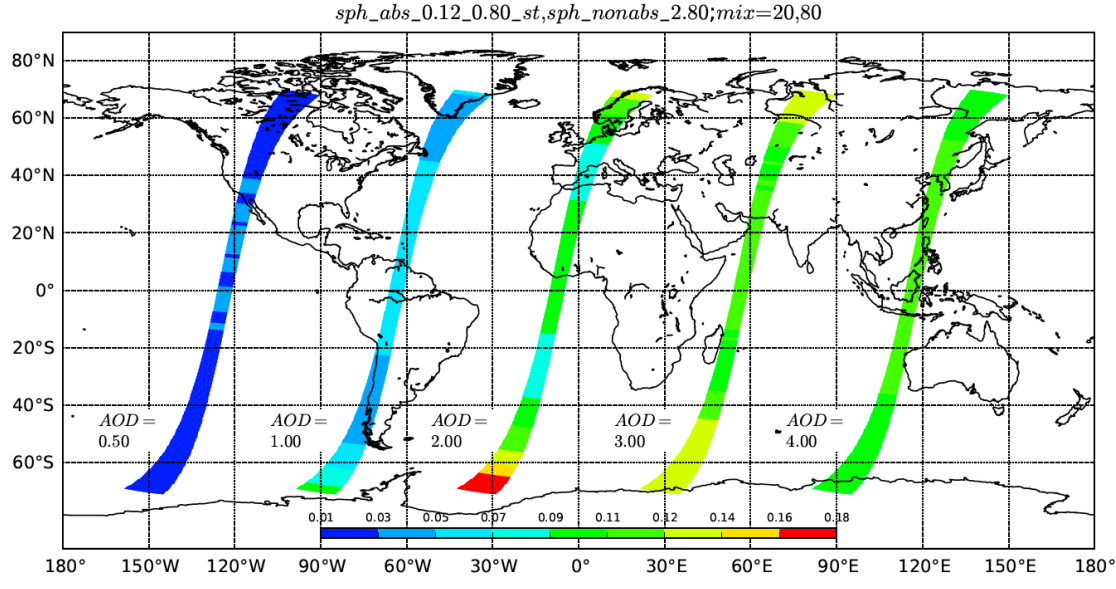

$$
\begin{gathered}
0.12 \text { SSA_0.80 } \\
+2.80 \mu \mathrm{m} \\
r_{e} \text { particles }
\end{gathered}
$$

- MLM overestimates AOD with spherical non-absorbing/absorbing particle mixtures 


\section{Impact of MLM; Globally, Absorbing Mixtures}

Median \% Deviation from Reference AOD, Chis $q_{M^{3} x^{3}} \leq 1.00 ; 2012-03-20 ;$ Orbit $=65179$, Path $=133$ sph_abs_0.12_0.75_st,sph_nonabs_2.80;mix $=80,20$

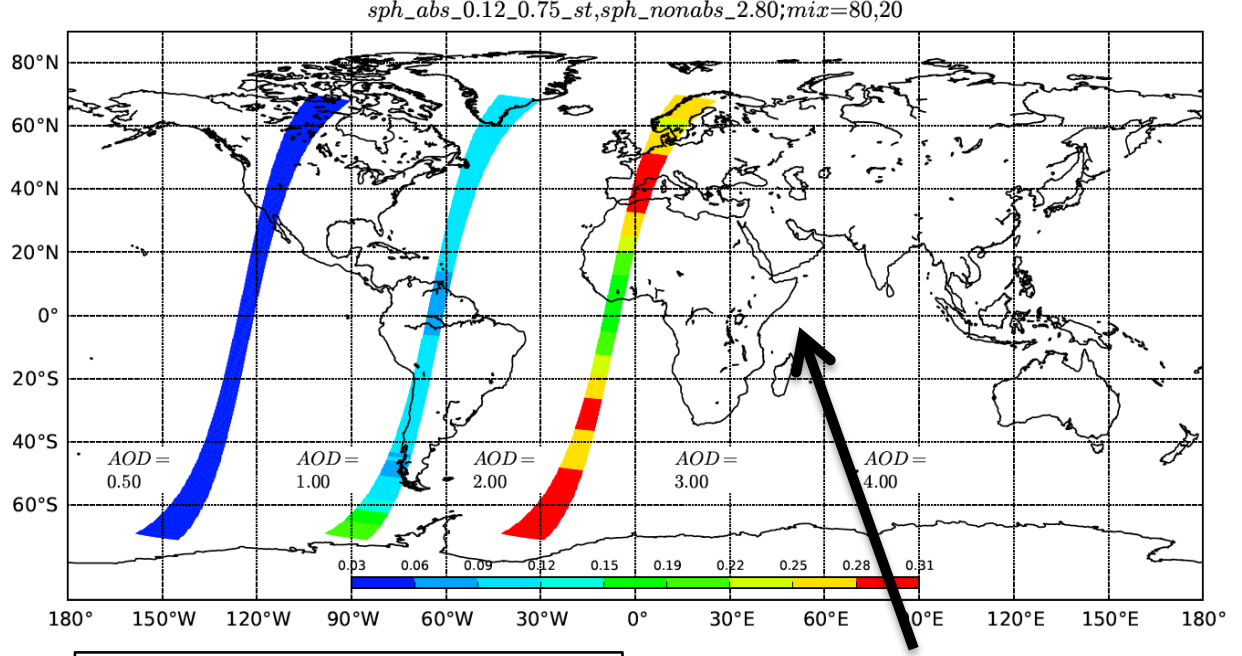

$<+31 \%$ Discrepancy $80 \%$ sph_abs_0.12_SSA-0.75 20\% sph_nonabs_2.80

$<+11 \%$ Discrepancy $20 \%$ sph_abs_0.12_SSA-0.75 $80 \%$ sph_nonabs_2.80
Median \% Deviation from Reference AOD, Chisq Max $^{3} \leq 1.00 ; 2012-03-20$; Orbit $=65179$, Path $=133$ $s p h \_a b s \_0.12 \_0.75 \_s t, s p h \_n o n a b s \_2.80 ; m i x=50,50$

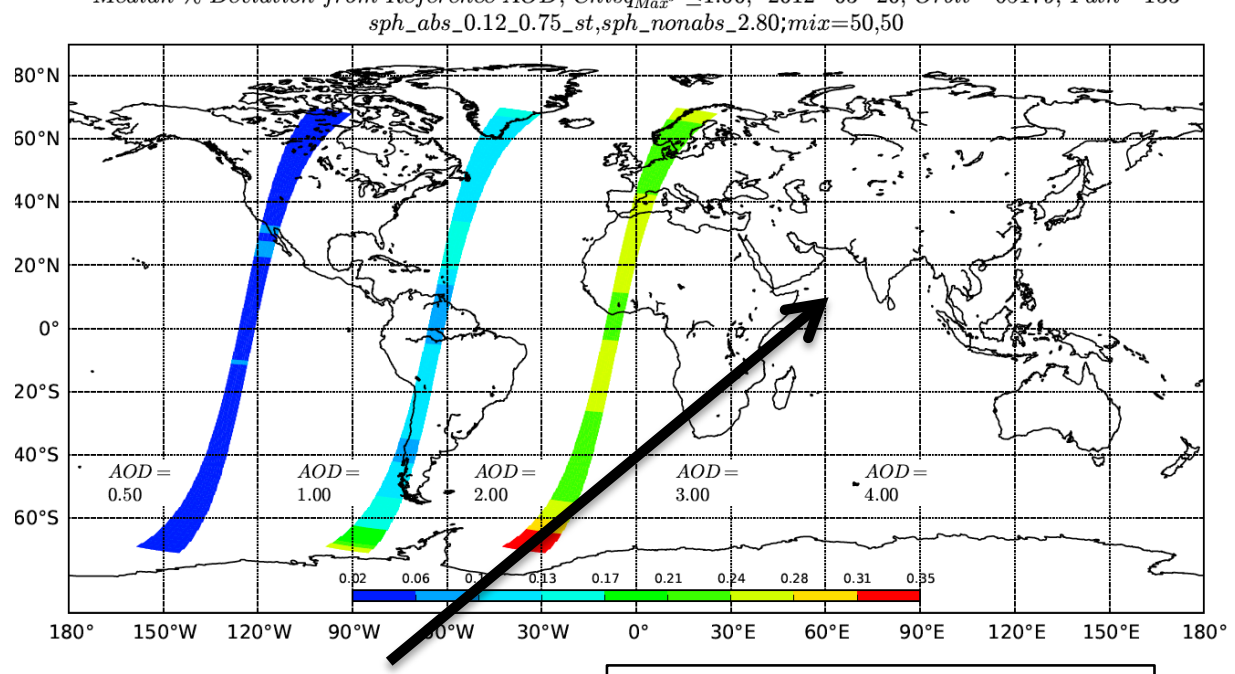

$<+28 \%$ Discrepancy

Note: At high AOD $\rightarrow$

No mixtures pass when MLM is used

Median \% Deviation from Reference AOD, Chisq (nx $^{3} \leq 1.00 ; 2012-03-20 ;$ Orbit $=65179$, Path $=133$ 50\% sph_abs_0.12_SSA-0.75 $50 \%$ sph_nonabs_2.80

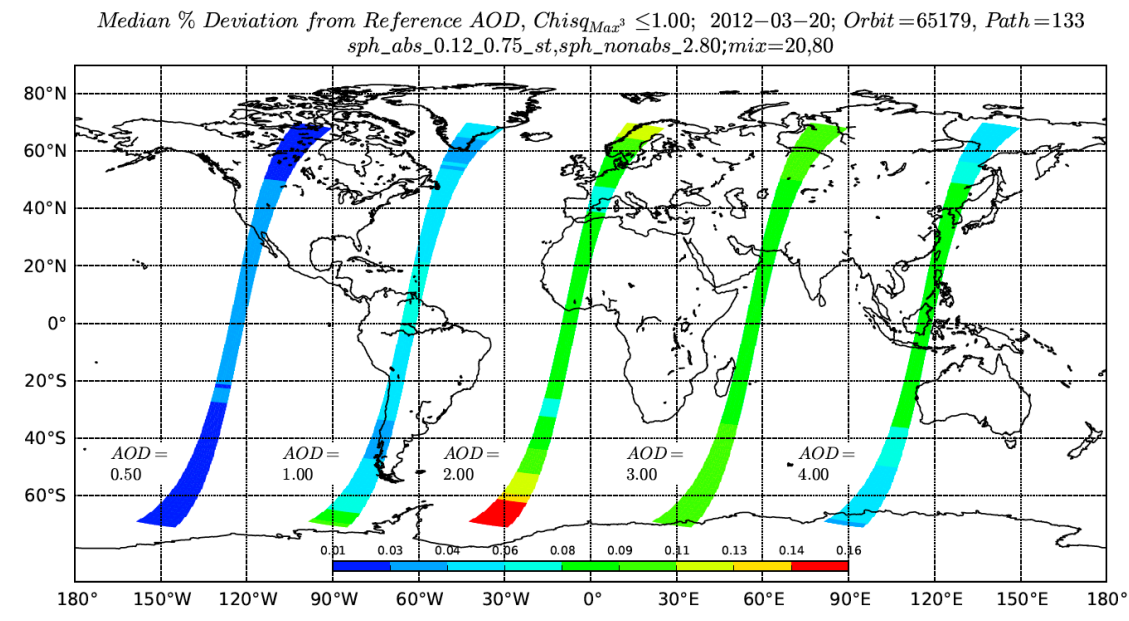

- MLM overestimates AOD with spherical non-absorbing/absorbing particle mixtures 


\section{Impact of MLM; Globally, Non-Spherical Mixtures}

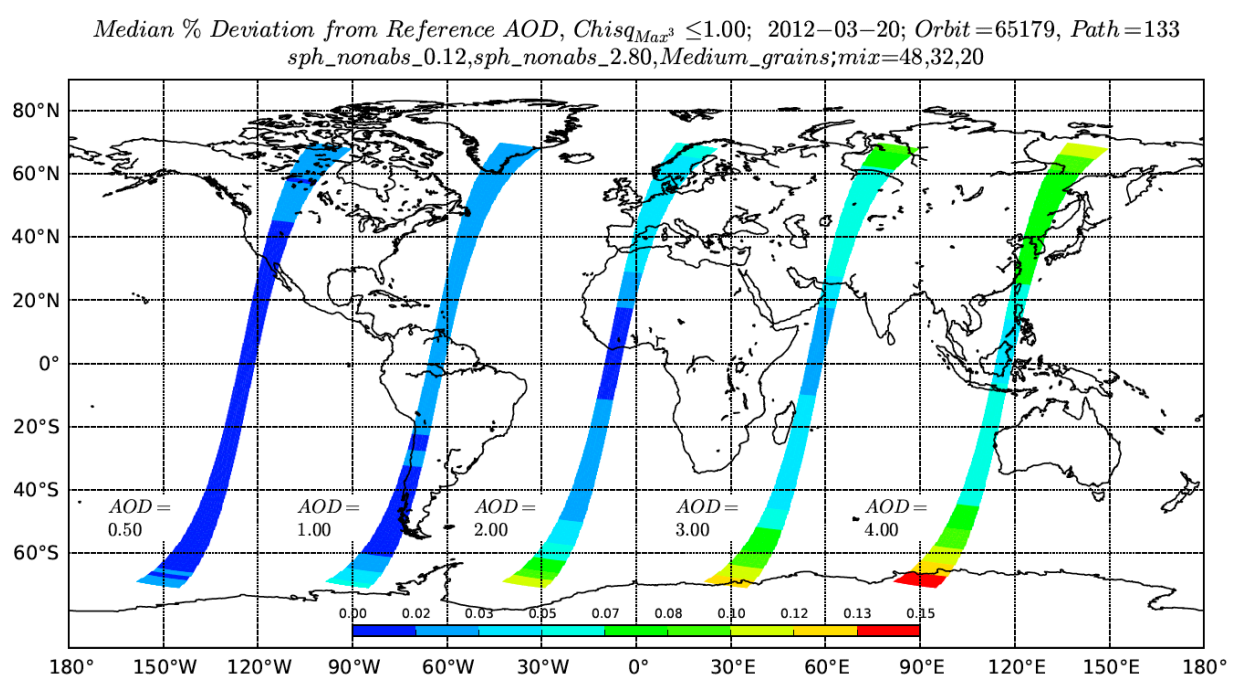

$<+10 \%$ Discrepancy, Mix \#52 48\% sph_nonabs_0.12 $32 \%$ sph_nonabs_2.80 20\% Medium Dust Grains

\section{$<+6 \%$ Discrepancy, Mix \#58} 24\%sph_nonabs_0.12 $16 \%$ sph_nonabs_2.80 $60 \%$ Medium Dust Grains

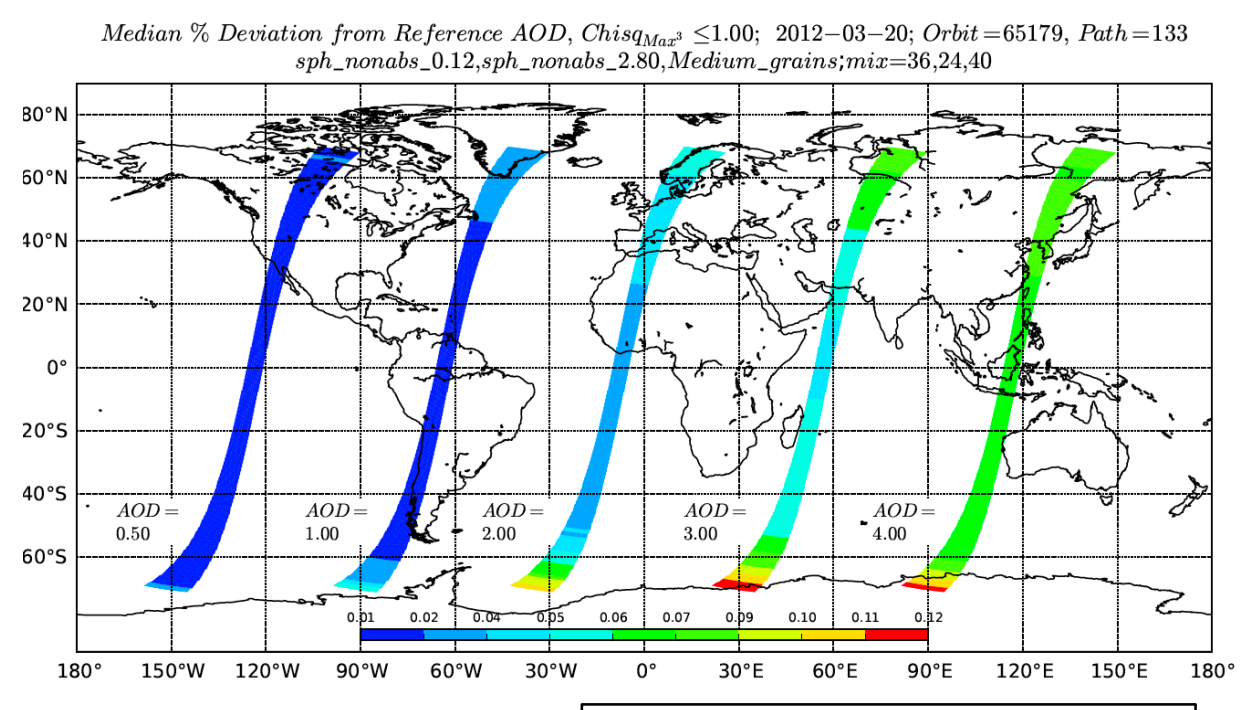

< +9\% Discrepancy, Mix \#55 $36 \%$ sph_nonabs_0.12 $24 \%$ sph_nonabs_2.80 40\% Medium Dust Grains

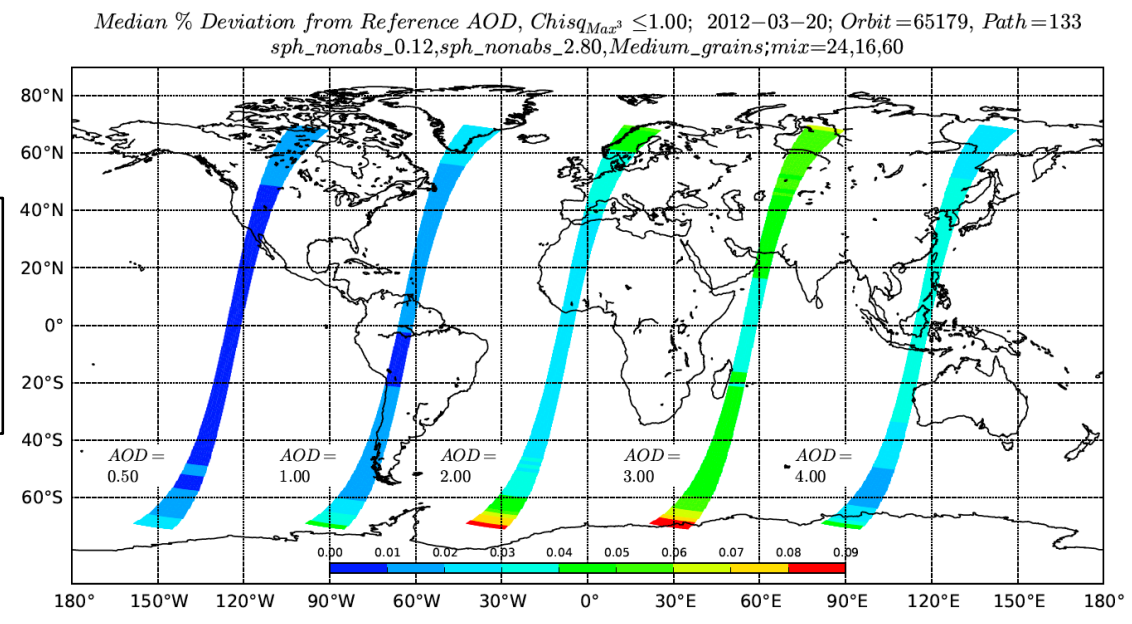

$0.12+2.80 \mu \mathrm{m}$ + dust grain $r_{e}$ particles

- Modified linear mixing overestimates AOD with non-spherical particle mixtures 


\section{Impact of MLM; Globally, Non-Spherical Mixtures}

Median \% Deviation from Reference AOD, Chisq Max $^{3} \leq 1.00 ; 2012-03-20 ;$ Orbit $=65179$, Path $=133$ sph_nonabs_0.12,Medium_grains,Coarse_spheroids; mix $=40,24,36$

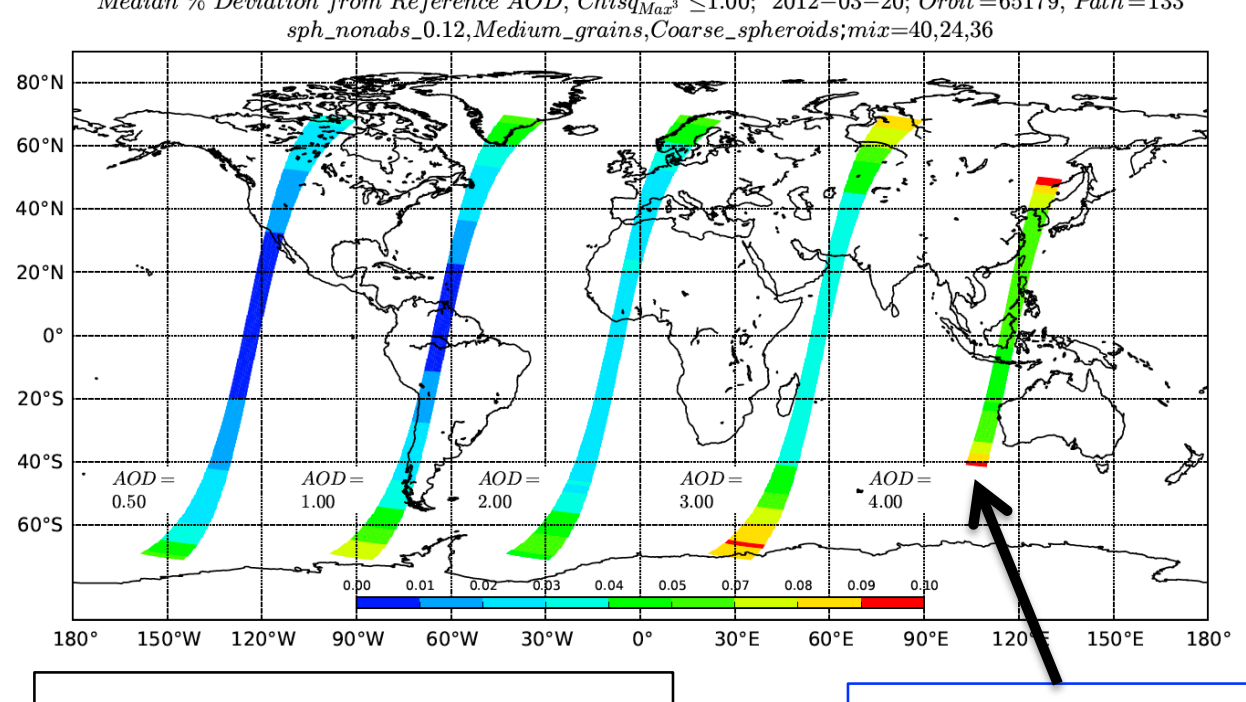

$<+10 \%$ Discrepancy, Mix \#65 40\% sph_nonabs_0.12 24\% Medium Dust Grains $36 \%$ Coarse Dust Spheroids

$<+9 \%$ Discrepancy, Mix \#73 0\% sph_nonabs_0.12 40\% Medium Dust Grains $60 \%$ Coarse Dust Spheroids

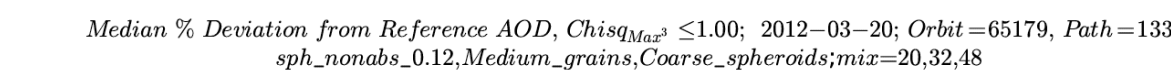

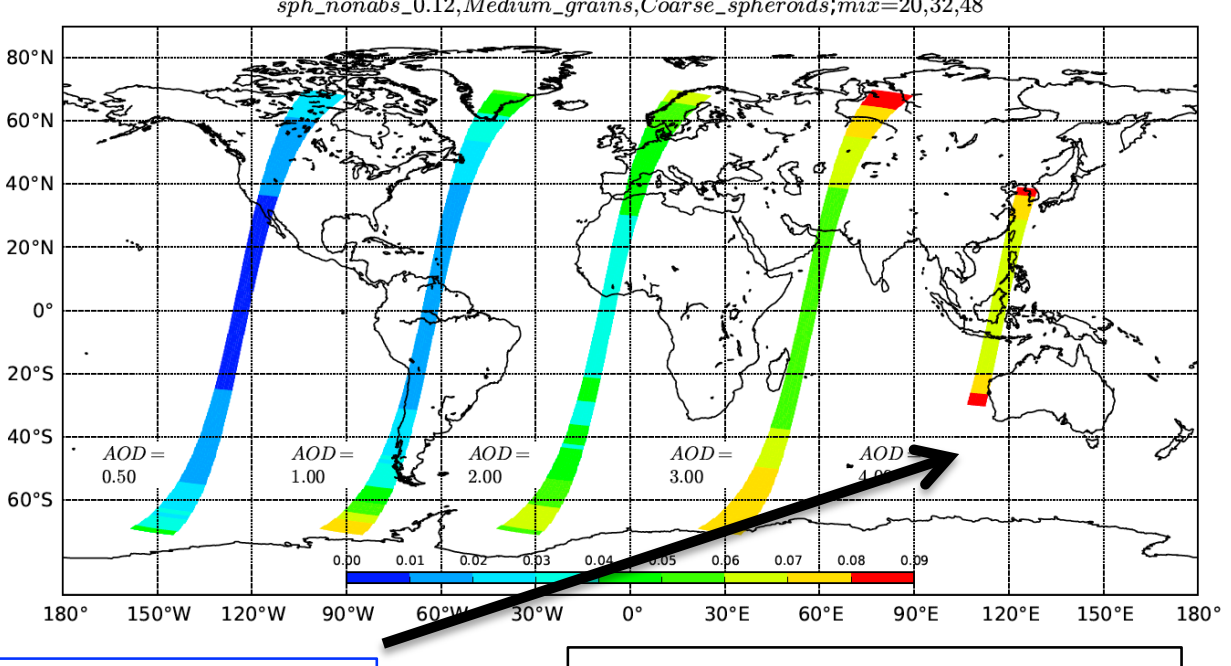

$<+9 \%$ Discrepancy, Mix \#69 20\% sph_nonabs_0.12 32\% Medium Dust Grains 48\% Coarse Dust Spheroids

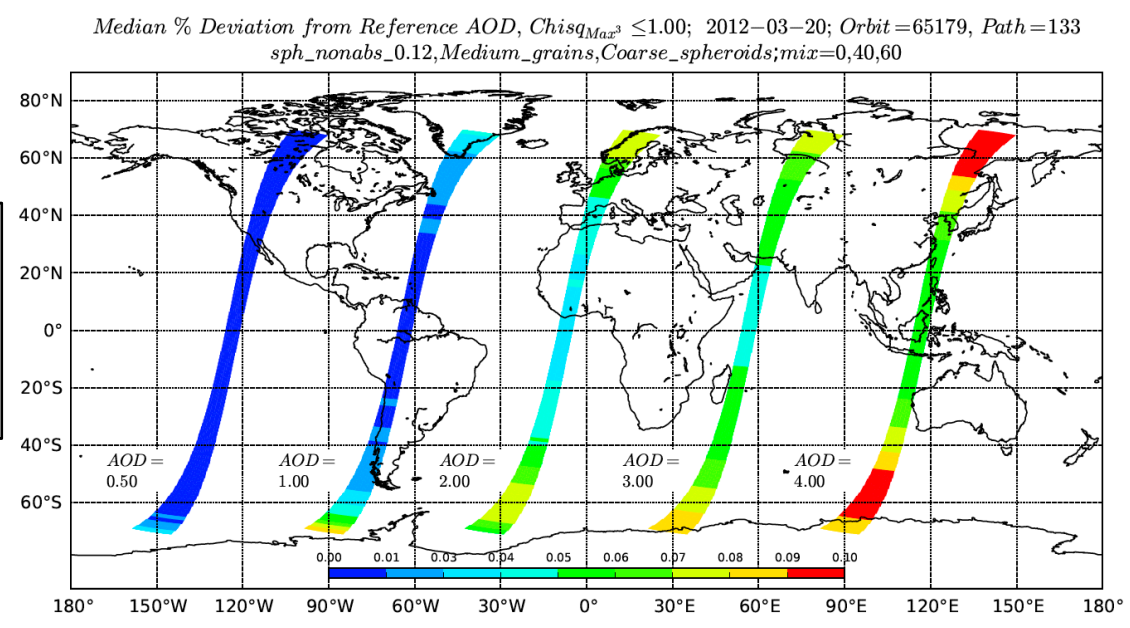

ote: At high lat \& high AOD $\rightarrow$ No mixtures pass when MLM is used

Median 7 Deciation from Reference AOD, Chis , $\leq 100$; 2012-03-20; Orbit=65179, Path $=133$

$0.12 \mu \mathrm{m}$
+ dust grain
+ dust spheroid
$r_{e}$ particles

- Modified linear mixing overestimates AOD with non-spherical particle mixtures 


\section{Linear Mixing (LM) \& Modified-Linear Mixing (MLM) Sensitivity Study Conclusions}

- For spherical non-absorbing mixtures (Mixtures \#1-30) [LM, Figures S6-S10]

-- Retrieved AOD values still fall within 0.05 or $20 \%$ AOD

-- $<5 \%$ bias for $A O D \leq 1.0$ in all cases

-- Larger particle size difference $\rightarrow$ larger AOD overestimate

So the largest bias is for [0.06 $+\mathbf{2 . 8 0} \mu \mathrm{m}]$ mixtures

-- The effect becomes more pronounced at high AODs (up to $18 \%$ for AOD > 2)

- For spherical absorbing mixtures, AOD is also overestimated [MLM, Figures S11-S14]

-- The mixtures considered here (\#31-50) perform reasonably well at low AODs

[sph_abs_0.12_SSA-0.80 or $90 \mathbf{\&}+\mathbf{2 . 8 0} \mu \mathrm{m}$ non-abs.]

$<10 \%$ bias for $A O D \leq 1.0$

-- Mixtures having larger fractions $2.80 \mu \mathrm{m}$ non-abs. particles (\#34-40 \& 44-49) can fall outside of 0.05 or $20 \%$ AOD for AOD $\geq 2.0$ for some geometries

-- Biases of 10-30\% for AOD >2.0, even when SSA $0.12 \mu \mathrm{m}=0.95$

- For non-spherical mixtures, AOD is also overestimated [MLM, Figures S15-S16]

-- But $<\mathbf{1 0 \%}$ bias for all non-spherical mixtures in the MISR V22 SA climatology 